\title{
Weak lensing study of 16 DAFT/FADA clusters: Substructures and filaments ${ }^{\star}$
}

\author{
Nicolas Martinet ${ }^{1,2}$, Douglas Clowe ${ }^{3}$, Florence Durret ${ }^{1}$, Christophe Adami $^{4}$, Ana Acebrón ${ }^{4}$, \\ Lorena Hernandez-García ${ }^{5}$, Isabel Márquez ${ }^{5}$, Loic Guennou ${ }^{6}$, Florian Sarron ${ }^{1}$, and Mel Ulmer ${ }^{7}$ \\ 1 Sorbonne Universités, UPMC Université Paris 6 et CNRS, UMR 7095, Institut d'Astrophysique de Paris, 98 bis Bd Arago, \\ 75014 Paris, France \\ e-mail: martinet@iap.fr \\ 2 Argelander-Institut für Astronomie, Universität Bonn, Auf dem Hügel 71, 53121 Bonn, Germany \\ 3 Department of Physics and Astronomy, Ohio University, 251B Clippinger Lab, Athens, OH 45701, USA \\ ${ }^{4}$ LAM, OAMP, Université Aix-Marseille \& CNRS, Pôle de l'Étoile, Site de Château Gombert, 38 rue Frédéric Joliot-Curie, \\ 13388 Marseille 13 Cedex, France \\ 5 Instituto de Astrofísica de Andalucía, CSIC, Glorieta de la Astronomía s/n, 18008 Granada, Spain \\ ${ }^{6}$ Institut d'Astrophysique Spatiale, CNRS, UMR8617, Université Paris-Sud 11, Bâtiment 121, 91405 Orsay, France \\ 7 Dept of Physics and Astronomy \& Center for Interdisciplinary Exploration and Research in Astrophysics (CIERA), Evanston, \\ IL 60208-2900, USA
}

Received 30 April 2015 / Accepted 15 March 2016

\begin{abstract}
While our current cosmological model places galaxy clusters at the nodes of a filament network (the cosmic web), we still struggle to detect these filaments at high redshifts. We perform a weak lensing study for a sample of 16 massive, medium-high redshift $(0.4<z<0.9)$ galaxy clusters from the DAFT/FADA survey, which are imaged in at least three optical bands with Subaru/SuprimeCam or CFHT/MegaCam. We estimate the cluster masses using an NFW fit to the shear profile measured in a KSB-like method, adding our contribution to the calibration of the observable-mass relation required for cluster abundance cosmological studies. We compute convergence maps and select structures within these maps, securing their detection with noise resampling techniques. Taking advantage of the large field of view of our data, we study cluster environment, adding information from galaxy density maps at the cluster redshift and from X-ray images when available. We find that clusters show a large variety of weak lensing maps at large scales and that they may all be embedded in filamentary structures at megaparsec scale. We classify these clusters in three categories according to the smoothness of their weak lensing contours and to the amount of substructures: relaxed ( $\sim 7 \%)$, past mergers $(\sim 21.5 \%)$, and recent or present mergers $(\sim 71.5 \%)$. The fraction of clusters undergoing merging events observationally supports the hierarchical scenario of cluster growth, and implies that massive clusters are strongly evolving at the studied redshifts. Finally, we report the detection of unusually elongated structures in CLJ0152, MACSJ0454, MACSJ0717, A851, BMW1226, MACSJ1621, and MS1621.
\end{abstract}

Key words. galaxies: clusters: general - gravitational lensing: weak - large-scale structure of Universe

\section{Introduction}

In cold dark matter (CDM) theories, our Universe can be represented as an ensemble of large-scale structures (LSSs) made of voids and galaxy clusters that are connected through filamentary structures (Bond et al. 1996). In this scenario, matter collapses into halos that then grow through accretion and merging with other halos. Galaxy clusters are the highest density structures resulting from this hierarchical formation. $N$-body simulations

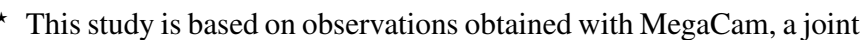
project of CFHT and CEA/IRFU, at the Canada-France-Hawaii Telescope (CFHT), which is operated by the National Research Council (NRC) of Canada, the Institut National des Sciences de l'Univers of the Centre National de la Recherche Scientifique (CNRS) of France, and the University of Hawaii. The study is also based on archive data collected at the Subaru Telescope, which is operated by the National Astronomical Observatory of Japan. This research made use of data obtained from the Chandra Data Archive provided by the Chandra X-ray Center (CXC) and data obtained from the XMM-Newton Data Archive provided by the XMM-Newton Science Archive (XSA).
}

(e.g., Millennium; Springel et al. 2005) and low redshift observations (e.g., SDSS; Tegmark et al. 2004) have confirmed this evolutionary scheme.

In this framework, galaxy clusters can be used to constrain cosmological models. Indeed, the distribution of clusters with mass and redshift contains information on the mentioned hierarchical formation scenario (e.g., Allen et al. 2011). The main challenge is to calibrate the so-called observable-mass relation that links true cluster masses to the mass proxy used in the survey. With its ability to be insensitive to the matter dynamical state, weak lensing (WL) appears as a major tool in determining the masses of galaxy clusters with sufficient precision to derive cosmological constraints. However, this technique requires a large amount of clusters and, therefore, more and more WL surveys with increasing numbers of clusters are conducted (e.g., Dahle et al. 2002; Cypriano et al. 2004; Clowe et al. 2006; Gavazzi \& Soucail 2007; Hoekstra 2007; Okabe et al. 2010; von der Linden et al. 2014; Hoekstra et al. 2015). In a similar idea, Martinet et al. (2015a) recently showed that counting shear 
peaks can constrain cosmological parameters almost as well as counting galaxy clusters, without requiring any knowledge of the observable-mass relation, but needing a large number of cosmological simulations.

As it directly traces the matter density, WL also allows us to study the LSSs of our Universe. However, the low density of filaments compared to clusters makes their detection difficult. Several studies pioneered in using WL to detect such structures in the vicinity of clusters either by reporting low significance detection or questioning previous claims of detection (e.g., Clowe et al. 1998; Kaiser et al. 1998; Gray et al. 2002; Gavazzi et al. 2004; Dietrich et al. 2005; Heymans et al. 2008; Dietrich et al. 2012). Massey et al. (2007b) found evidence for a cosmic network of filaments in the COSMOS field galaxy survey. Mead et al. (2010) used the Millennium Simulation (Springel et al. 2005) to test the ability of various WL techniques to detect nearby cluster filaments, and concluded that background galaxy density is key to filament detection. Future space-based missions are likely to detect many filaments, but today, the narrow field of view of the Advanced Camera for Surveys (ACS) on the Hubble Space Telescope (HST) does not allow such detection in a simple way. In this context, deep ground-based imaging can be very efficient as it often has a much wider field of view and offers the possibility of covering clusters and their vicinity in a single image with Subaru/SuprimeCam or CFHT/Megacam. Recently, Jauzac et al. (2012) reported the first WL detection of a $z=0.54$ cluster with a filament, MACSJ0717.5+3745 based on a mosaic of HST/ACS images. This detection was latter confirmed by Medezinski et al. (2013) from a Subaru/Suprime-Cam WL analysis.

In this paper, we present the WL analysis of 16 clusters from the Dark energy American French Team (DAFT, FADA in French ) survey. All are medium-high redshift $(0.4 \leq z \leq 0.9)$ massive $\left(M \geq 2 \times 10^{14} M_{\odot}\right)$ clusters of galaxies selected through their X-ray luminosities. This sample is comparable to other $\mathrm{X}$-ray selected cluster studies such as LOCUSS at $0.15 \leq z \leq 0.3$ (Okabe et al. 2010), Weighting the Giants at $0.15 \leq z \leq 0.7$ (von der Linden et al. 2014), and CCCP at $0.15 \leq z \leq 0.55$ (Hoekstra 2007; Hoekstra et al. 2015) with a slightly higher redshift, but with fewer clusters than the mentioned surveys that contain 30, 51, and 50 galaxy clusters, respectively. Apart from estimating cluster masses, we take advantage of the large field of view of our images (eight CFHT/Megacam images with $1 \mathrm{deg}^{2}$ field of view and seven Subaru/Suprime-Cam images with $34 \times 27 \mathrm{arcmin}^{2}$ field of view; one of the Subaru images contains two clusters) to investigate galaxy cluster environments. In particular, we report the WL detection of several elongated structures that might correspond to filaments.

This paper is structured as follows. Section 2 describes our data set, Sect. 3 presents the shear measurement we apply in detail, and Sect. 4 explains the mass reconstruction process. In Sect. 5, we estimate the cluster masses and in Sect. 6 we focus on the environment of clusters: substructures, mergers, and filaments. We conclude in Sect. 7. Throughout the paper, we use a fiducial flat $\Lambda$ CDM cosmology with $\Omega_{\mathrm{M}}=0.3, \Omega_{\Lambda}=0.7$, and $H_{0}=70 \mathrm{~km} \mathrm{Mpc}^{-1} \mathrm{~s}^{-1}$. All displayed distances are comoving.

\section{Data}

\subsection{DAFT/FADA}

DAFT/FADA is a survey of $\sim 90$ medium-high-redshift $(0.4 \leq$ $z \leq 0.9)$ massive $\left(M \geq 2 \times 10^{14} M_{\odot}\right)$ clusters of galaxies selected through their X-ray luminosities. All of the clusters have
HST imaging available with either WFPC2 or ACS cameras. We also gathered multiband optical and near-infrared ground-based imaging, using 4-m class telescopes for most of the sample. This data set allows us to accurately measure the ellipticity of galaxies from space and their photometric redshifts (hereafter photo- $z$ ) from the ground. The main goals of the survey are to form a comprehensive database to study galaxy clusters and their evolution, and to test cosmological constraints geometrically by means of weak lensing tomography. Several steps have been made toward the achievement of these two goals, and the current status of the survey, with a list of refereed publications, can be found at http: //cesam. lam. fr/DAFT/project.php.

Among other papers, Murphy et al. (2015) performed a WL analysis of HST/ACS mosaic imaging data of ten massive, highredshift $(z>0.5)$ DAFT/FADA galaxy clusters. Using the photo$z$ s calculated by Guennou et al. (2010), Murphy et al. explored their use for background galaxy discrimination. Our team is currently increasing this small sample of HST/ACS shear measurements to a larger number of clusters and also aims at combining ground-based and space-based shear catalogs to build a shear analysis that is both deep in the cluster central region and extended on larger scales. This will serve as the reference catalog to perform weak lensing tomography with clusters (WLTC) as described in Jain \& Taylor (2003).

\subsection{This study}

In this study, we focus on 16 galaxy clusters for which we have Subaru/Suprime-Cam or CFHT/Megacam wide field images for at least three optical bands among the $v, r, i$, and $z$ bands. Having three bands is mandatory to be able to perform a color-color cut to remove foreground galaxies that dilute the lensing signal. The shear measurements are performed in the $r$ or $i$ bands depending on the image seeing. This choice is made to maximize the number of source galaxies as these bands are the deepest optical bands. The use of Suprime-Cam $\left(34 \times 27 \operatorname{arcmin}^{2}\right.$ field $)$ and Megacam $\left(1 \times 1 \mathrm{deg}^{2}\right.$ field) imaging allows us to study clusters within their virial radius and also to see how they interplay with the surrounding LSS at the selected redshifts $(0.4 \leq z \leq 0.9)$. These fields of view are much wider than what can be achieved from current space telescopes, as the HST/ACS field of view is only $3.4 \times 3.4 \mathrm{arcmin}^{2}$. Besides, the Megacam and SuprimeCam cameras present rather stable point spread functions (PSFs) and contain a large number of stars within each pointing allowing us to accurately estimate the PSF distortion due to the instrument and atmospheric biases. A list of the data for each cluster can be found in Table 1 .

Some of the clusters from the present study have been analyzed in previous DAFT/FADA papers. Guennou et al. (2014) derived X-ray luminosities and temperatures for 12 out of these 16 clusters. A comparison of WL and X-ray total masses is performed in Sect. 5.2. Guennou et al. (2014) also searched for substructures using both X-ray data and optical galaxy spectroscopy. Martinet et al. (2015b) studied the optical emission of galaxy clusters and measured the galaxy luminosity functions (GLFs) for seven out of these 16 clusters. We indicate which study included each cluster in Table 1.

With the present DM study, we will gain a full understanding of the matter content of a sample of galaxy clusters: the DM halo, the X-ray intracluster medium (ICM), and the stars contained in galaxies. Even if we do not include all the clusters in each analysis, we will have a general knowledge of cluster behaviors as observed through WL, X-rays, and optical. 
Table 1. Data used in this study.

\begin{tabular}{lcccccccc}
\hline \hline Cluster & RA & Dec & $z$ & Instrument & Filters & Seeing & G14 & M15 \\
\hline XDCScmJ032903 & 032902.81 & +025625.18 & 0.4122 & CFHT/Megacam & $r+(v, i)$ & $0.73^{\prime \prime}$ & $\mathrm{Y}$ & $\mathrm{Y}$ \\
MACSJ0454.1-0300 & 045410.92 & -030107.14 & 0.5377 & CFHT/Megacam & $r+(v, z)$ & $0.76^{\prime \prime}$ & $\mathrm{Y}$ & $\mathrm{Y}$ \\
ABELL0851 & 094256.64 & +465921.91 & 0.4069 & CFHT/Megacam & $i+(v, z)$ & $0.80^{\prime \prime}$ & $\mathrm{Y}$ & $\mathrm{Y}$ \\
LCDCS0829 & 134731.99 & -114542.01 & 0.4510 & CFHT/Megacam & $r+(v, i)$ & $0.83^{\prime \prime}$ & $\mathrm{Y}$ & $\mathrm{Y}$ \\
MS1621.5+2640 & 162335.50 & +263413.00 & 0.4260 & CFHT/Megacam & $r+(v, i)$ & $0.65^{\prime \prime}$ & $\mathrm{Y}$ & $\mathrm{N}$ \\
OC02J1701+6412 & 170122.60 & +641409.00 & 0.4530 & CFHT/Megacam & $r+(i, v)$ & $0.73^{\prime \prime}$ & $\mathrm{N}$ & $\mathrm{N}$ \\
NEP0200 & 175719.39 & +663131.00 & 0.6909 & CFHT/Megacam & $i+(v, r)$ & $0.97^{\prime \prime}$ & $\mathrm{N}$ & $\mathrm{N}$ \\
RXJ2328.8+1453 & 232849.90 & +145312.01 & 0.4970 & CFHT/Megacam & $r+(v, i)$ & $0.70^{\prime \prime}$ & $\mathrm{Y}$ & $\mathrm{N}$ \\
CLJ0152.7-1357 & 015240.99 & -135745.00 & 0.8310 & Subaru/Suprime-Cam & $r+(v, z)$ & $0.70^{\prime \prime}$ & $\mathrm{Y}$ & $\mathrm{Y}$ \\
MACSJ0717.5+3745 & 071733.79 & +374520.01 & 0.5458 & Subaru/Suprime-Cam & $r+(v, z)$ & $0.69^{\prime \prime}$ & $\mathrm{N}$ & $\mathrm{N}$ \\
BMW-HRIJ122657 & 122658.00 & +333254.09 & 0.8900 & Subaru/Suprime-Cam & $r+(i, z)$ & $0.80^{\prime \prime}$ & $\mathrm{Y}$ & $\mathrm{Y}$ \\
MACSJ1423.8+2404 & 142348.29 & +240446.99 & 0.5450 & Subaru/Suprime-Cam & $i+(v, r)$ & $0.88^{\prime \prime}$ & $\mathrm{Y}$ & $\mathrm{Y}$ \\
MACSJ1621.4+3810 & 162123.99 & +381001.99 & 0.4650 & Subaru/Suprime-Cam & $i+(v, r)$ & $0.62^{\prime \prime}$ & $\mathrm{N}$ & $\mathrm{Y}$ \\
RXJ1716.4+6708 & 171649.60 & +670830.01 & 0.8130 & Subaru/Suprime-Cam & $r+(v, z)$ & $0.63^{\prime \prime}$ & $\mathrm{Y} / \mathrm{N}$ & $\mathrm{N}$ \\
CXOSEXSIJ205617* & 205617.16 & -044155.10 & 0.6002 & Subaru/Suprime-Cam & $r+(v, i)$ & $0.61^{\prime \prime}$ & $\mathrm{Y}$ & $\mathrm{N}$ \\
MS2053.7-0449* & 205622.37 & -043743.42 & 0.5830 & Subaru/Suprime-Cam & $r+(v, i)$ & $0.61^{\prime \prime}$ & $\mathrm{Y}$ & $\mathrm{N}$ \\
\hline
\end{tabular}

Notes. The different columns correspond to \#1: cluster ID; \#2: right ascension; \#3: declination; \#4: redshift; \#5: telescope/camera; \#6: filters, we give first the band on which we perform shape measurements and in parentheses the two other bands used for the color-color cut; and \#7: seeing for the band on which we perform shape measurements. Columns \#8 (G14) and \#9 (M15) show whether the cluster was studied in Guennou et al. (2014) or Martinet et al. (2015b). In the first case, we know if it presents substructures based on X-ray images, and in most cases, on optical galaxy spectroscopy as well. For RX_J1716.4+6708, we have spectroscopy but no XMM image. In the second, we have photo-zs in the inner part, and in most cases, an optical galaxy luminosity function for the cluster. Hereafter, we use abridged names. ${ }^{(*)}$ CXOSEXSI_J205617 and MS_2053.7-0449 are on the same image.

\subsection{Image reduction}

The Subaru and CFHT data presented here are archive data, either from previous studies or from the early phases of DAFT/FADA.

The CFHT/Megacam data were reduced by the TERAPIX team at the Institut d'Astrophysique de Paris via the astromatic software ${ }^{1}$. Sources are detected with SExtractor (Bertin \& Arnouts 1996) and an astrometric solution is found using SCAMP (Bertin 2006). The stacking of the dithered exposures is then performed via SWarp (Bertin et al. 2002). We measure the seeing by fitting a Gaussian surface brightness profile to the bright stars of the image with PSFEx (Bertin 2011).

The images obtained with the Subaru telescope and SuprimeCam were retrieved in raw form from the SMOKA archive ${ }^{2}$ together with the calibration files (bias and sky flat-field exposures), except the images of MACSJ0717, which were taken from Medezinski et al. (2013). These images were reduced in the usual way, by subtracting an average bias and dividing by the normalized flat field in each filter exactly in the same way as the images we observed ourselves. The reduced images were then calibrated astrometrically using the SCAMP and SWarp tools and combined for each filter. The photometric calibration was made in priority with SDSS catalogs when available in the field and in the corresponding band. If not available, we used the observed standard stars.

\section{Shear measurement}

The main idea of lensing is to reconstruct the mass distribution of a foreground object, designated as the lens, through the deflection it induces on the background object light, namely galaxy

\footnotetext{
1 http://www .astromatic.net/

2 http://smoka.nao.ac.jp/
}

sources. In the WL regime, the deflection is smaller than the typical intrinsic ellipticity of a galaxy (on the order of the percent), so that we must take the mean of many shear measurements from individual galaxies to reach a high signal-to-noise $(\mathrm{S} / \mathrm{N})$ detection of the shear. For a complete description of this phenomenon, check, for example, the review by Bartelmann \& Schneider (2001). The main difficulty of the method is to take into account all the galaxy shape distortions that are not due to the shear signal, such as atmospheric variations and instrumental biases. To correct for these biases, we apply a KSB+ method, initially proposed by Kaiser, Squires, and Broadhurst (Kaiser et al. 1995), and later refined by Luppino \& Kaiser (1997) and Hoekstra et al. (1998). The KSB method suits shear measurements in cluster fields well, as assessed by the various large surveys choosing this technique (Okabe et al. 2010; von der Linden et al. 2014; Hoekstra et al. 2015). In addition, this method has been accurately tested on simulated images such as the STEP2 simulations by Massey et al. (2007a). Most of the WL reduction presented here is similar to the technique applied in Clowe et al. (2012).

We first detect objects using SExtractor and clean the catalog from spurious detections (Sect. 3.1). We separate stars from galaxies and measure the instrument PSF variation on stars (Sect. 3.2) using the IMCAT software (Kaiser $(2011)^{3}$ ) with some additional developments. We correct galaxy shapes for the PSF anisotropies to obtain an individual object shear cata$\log$ (Sect. 3.3). We then smooth the shear measurement noise (Sect. 3.4) and correct for the methodology biases by testing our reduction on the STEP2 (Massey et al. 2007a) shear simulations (Sect. 3.5).

\subsection{Source detection}

We use SExtractor to detect objects and measure their photometry in our images. In most cases, the precise alignments of the

\footnotetext{
3 http://www.ifa.hawaii.edu/ kaiser/imcat/
} 
three bands are sufficient to enable a detection in double image mode. We then perform the initial detection in the band used for shape measurements and detect objects in the same apertures and positions in the two other images. For some Subaru images, we did not manage to align the images from all three bands precisely. The detection is then performed separately in each band and measurements are associated with those in the band on which the ellipticity is measured. This cross correlation is done through a minimization of matched object distances with a 2 arcsec limit. We detect all objects that lie on at least three pixels above 1.5 times the sky background after convolving the surface brightness profile with a Gaussian kernel of $7 \times 7$ pixel size and 3 pixel FWHM. We use 32 deblending subthresholds with a deblending contrast close to zero in order to remove most of the possible blended objects that would have a modified shape. Object magnitudes are measured with the MAG_AUTO keyword.

We then compute the $\mathrm{S} / \mathrm{N}$ of each object using the getsig IMCAT tool. This command convolves the object surface brightness profile with a Gaussian filter of increasing smoothing radius $r_{\mathrm{g}}$ and selects the value of $r_{\mathrm{g}}$ that maximizes the $\mathrm{S} / \mathrm{N}$. At the same time, we obtain the best $\mathrm{S} / \mathrm{N}$ for the object and an estimate of its size with the $r_{\mathrm{g}}$ parameter. The local background is computed by fitting a mean sky level and a 2-d linear slope of the sky brightness in an annulus centered on the object, ignoring all the pixels within $3 r_{\mathrm{g}}$ of any object to avoid contamination. Once this accurate $\mathrm{S} / \mathrm{N}$ is computed, we remove all objects with $\mathrm{S} / \mathrm{N}$ lower than 10.

We measure the 1 st to 4 th order of the surface brightness profile of each object in a circular aperture of size $3 r_{\mathrm{g}}$ using a Gaussian weighting with $\sigma=r_{\mathrm{g}}$, through the getshapes IMCAT command. We reject objects for which the first moment of the surface brightness profile does not coincide within one pixel with the object peak position as detected by SExtractor. We adjust the position of the remaining objects to the first moment of the surface brightness profile that represents a subpixel estimate of the object peak position and remeasure the object shape centered on this new position.

We then apply a series of cuts to remove likely spurious detections. We first remove all objects that have a smaller size than the instrument PSF, i.e., those that have a radius $r_{\mathrm{g}}$ smaller than the minimum radius of stars, selected in a magnitude versus $r_{\mathrm{g}}$ diagram. We also remove all objects located at less than 20 pixels from the image edges to avoid measuring truncated objects. Finally, we remove bad pixel detection and only keep objects that do not have any neighbor within 10 pixels of their center.

This catalog is then separated between stars and galaxies in a half-light radius $r_{\mathrm{h}}$ versus magnitude plot, as shown in Fig. 1. Stars are selected as objects lying on the constant radius sequence and with appropriate magnitudes. This magnitude range is set by hand to avoid saturated stars and objects that are too faint. Galaxies are selected as all objects larger than the star sequence at the same magnitude, excluding the saturated objects that can be seen in the bright part of the diagram.

\subsection{Point spread function measurement}

The PSF of a given image represents the response of the instrument to a point-like source in the conditions of observation. The variations of the PSF across the image are due to the instrument characteristics and weather conditions. CFHT/Megacam and Subaru/Suprime-Cam have rather stable PSFs suitable for WL. Having a good seeing also diminishes the PSF correction that we need to apply. As stars are point-like sources, they are suitable for measuring the PSF of an image. The large field of

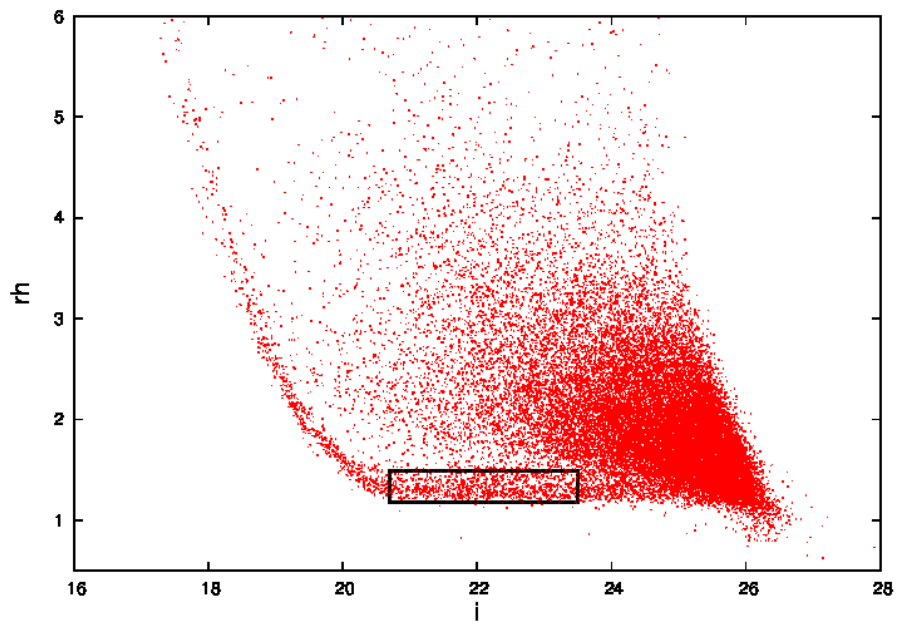

Fig. 1. Half-light radius $r_{\mathrm{h}}$ (in pixels) versus $i$-band magnitude diagram for MACSJ1621. Red dots are catalog objects. The star selection is represented by the black polygon. The sequence of saturated stars on the left side is removed and all remaining objects above the star sequence are considered galaxies.

view of our images enables us to have enough stars in a single frame to correct for the PSF anisotropies, in constrast to smaller field-of-view cameras that often require the use of stars across several images.

A general image distortion can be expressed by the two following quantities: the smear polarizability tensor $\boldsymbol{P}^{\mathrm{sm}}$ that describes the object response to the PSF anisotropy and the shear polarizability tensor $\boldsymbol{P}^{\text {sh }}$ that describes its response to the shear. These two tensors are measured from the 0th, 2nd, and 4th order moments of an object surface brightness distribution; see Kaiser et al. (1995) and Hoekstra et al. (1998) for the expression of these tensors. The ellipticity $\boldsymbol{e}$, is estimated from the 2 nd order moments of this distribution. In the next subsection, we use the following quantities, as measured on stars, to infer the true shape of galaxies: $\boldsymbol{P}_{\text {star }}^{\text {sm }}, \boldsymbol{P}_{\text {star }}^{\text {sh }}$, and $\boldsymbol{e}_{\text {star }}$.

Before measuring those quantities, we refine the star cata$\log$ to the cleanest objects. We first remove all objects that are closer than 40 pixels to any other object. We then fit the star ellipticities with a two-dimensional (2D) polynomial of the 6th order and generate modeled ellipticities at each object position using this polynomial. Objects that have a measured ellipticity differing by more than 0.05 from their modeled ellipticity are rejected. This step is repeated three times and permits us to remove galaxies that might have been considered stars. We chose an ellipticity cut at 0.05 as we found that it removes objects that are mainly out of the whole sample ellipticity distribution. Finally, a visual inspection is carried out to remove all remaining objects that could still suffer from blending issues or being close to saturated stars. The final catalogs contain $\sim 1000$ and $\sim 3000$ stars on average for Subaru and Megacam images, respectively, leading to an average star density of $1.0 \mathrm{arcmin}^{-2}$ and $0.8 \mathrm{arcmin}^{-2}$ for Subaru and Megacam, respectively.

Star shapes are measured using the getshapes IMCAT tool. As $\boldsymbol{P}^{\mathrm{sm}}$ and $\boldsymbol{P}^{\mathrm{sh}}$ depend on object sizes, we have to measure them for various sets of weighting radii. Hence, we compute a series of tensors for each $r_{\mathrm{g}}$ between 1 and 10 pixels with a step of 0.5 pixels, so that we can use the tensors corresponding to the galaxy radius when correcting for the PSF. Final quantities are fitted by 6th-order 2D polynomials as a function of position in order to have continuous functions defined at every point of the image. Here we chose to measure the PSF over the entire image, 
using a high order polynomial fit. However, in the case of large field-of-view images, one could also divide the frame into several small patches, and fit the PSF in each tile with a lower order polynomial. While the second approach is used in various studies (Okabe et al. 2010; Umetsu et al. 2011), von der Linden et al. (2014) applied and validated the first approach in the case of Subaru/Suprime-Cam images. For the CFHT/Megacam data, while fitting the PSF on each chip, Hoekstra (2007) found negligible discontinuities in the PSF anisotropy between chips. Following, for example, Massey et al. (2005), we compute the autocorrelation function of star ellipticities before and after the PSF correction and the cross-correlation function between galaxy shear and star ellipticities in Appendix A, thereby validating our PSF correction.

\subsection{Point spread function correction}

In the absence of noise, the shear of a background galaxy $\left(\boldsymbol{g}_{\mathrm{gal}}\right)$ can be computed from the following equation:

$\boldsymbol{g}_{\mathrm{gal}}=\left(\boldsymbol{P}_{\mathrm{gal}}^{\mathrm{g}}\right)^{-1} \delta \boldsymbol{e}_{\mathrm{gal}}$,

where $\boldsymbol{P}_{\text {gal }}^{\mathrm{g}}$ is the shear susceptibility tensor defined in Eq. (2) and $\boldsymbol{\delta} \boldsymbol{e}_{\text {gal }}$ is the apparent change in ellipticity described in Eq. (3). In Eq. (1) we neglect the intrinsic ellipticity that should be subtracted to the apparent ellipticity change $\left(\boldsymbol{\delta} \boldsymbol{e}_{\mathrm{gal}}\right)$. This is true if a sufficient number of galaxies is taken into account; as the galaxies are randomly oriented, the intrinsic ellipticity is null on average.

The shear susceptibility tensor represents the PSF corrected distortion, i.e., only due to the shear. We define it as in Luppino \& Kaiser (1997), i.e.,

$\boldsymbol{P}_{\text {gal }}^{\mathrm{g}}=\boldsymbol{P}_{\text {gal }}^{\mathrm{sh}}-\boldsymbol{P}_{\text {star }}^{\mathrm{sh}}\left(\boldsymbol{P}_{\text {star }}^{\mathrm{sm}}\right)^{-1} \boldsymbol{P}_{\text {gal }}^{\mathrm{sm}}$,

where the gal $_{\text {index is for tensors measured on galaxies and }}$ star for tensors measured on stars. The apparent change in ellipticity is:

$\boldsymbol{\delta} \boldsymbol{e}_{\text {gal }}=\boldsymbol{e}_{\text {gal }}-\boldsymbol{P}_{\text {gal }}^{\mathrm{sm}}\left(\boldsymbol{P}_{\text {star }}^{\mathrm{sm}}\right)^{-1} \boldsymbol{e}_{\text {star }}$,

where $\boldsymbol{e}$ represents the object ellipticity. In order to compute a galaxy shear, we then need to measure its ellipticity vector, smear polarizability, and shear polarizability tensors. This is again peformed with the getshapes tool. We also generate the star quantities corresponding to each galaxy radius $r_{\mathrm{g}}$ using the polynomials computed in the last section.

Prior to measuring the shape of galaxies, we reject QSOs and cosmic rays by removing objects that lie away from the principal sequence in a maximum flux versus magnitude diagram. We also remove objects in regions where the sky level is too bright to avoid star diffraction halos. We restrict our catalogs to objects larger than 1.5 times the PSF size, defined as the minimum star radius $r_{\mathrm{g}}$, deleting objects on which the PSF deconvolution could be too noisy. Finally, we visually inspect the images to remove any object close to saturated stars or reduction artifacts that could have survived our previous cleaning.

\subsection{Noise smoothing and coaddition}

The individual shear values are noisy owing to sky noise in the measurements of the higher order moments of the light distribution of objects. As these moments are subtracted from one another when computing the shear polarizibility tensor, the final signal value is reduced while the noise increases. We then
Table 2. Multiplicative $(m)$ and additive $(c)$ shear biases derived from applying our WL reduction pipeline to the STEP2 simulations with a Subaru PSF and a seeing of $0.8^{\prime \prime}$ (PSF C).

\begin{tabular}{ccc}
\hline \hline & $m$ & $c$ \\
\hline$\gamma_{1}$ & $-0.053 \pm 0.021$ & $0.004 \pm 0.001$ \\
$\gamma_{2}$ & $-0.021 \pm 0.030$ & $0.001 \pm 0.001$ \\
\hline
\end{tabular}

Notes. See Eq. (4) and text for details.

have to smooth the noise in the shear polarizibility tensor measurement to avoid it dominating the shear measurement, using its distribution across the image. We fit each component of the shear polarizability tensor $\boldsymbol{P}_{\text {gal }}^{\mathrm{g}}$ as a function of one component of the ellipticity and object size $r_{\mathrm{g}}$ by a 4 th order 2D polynomial. We chose a 4 th order polynomial after testing several orders, as we found that this polynomial minimized the noise. Also, we find that the shear polarizability tensor weakly depends on the ellipticity but is more sensitive to the object size. We then use this modeled tensor to regenerate the shear values of each object following Eq. (1). We note that this step removes the noise that would cause negative values of the shear polarizability tensor. We verify that after this fitting procedure, we have no $\boldsymbol{P}_{\text {gal }}^{\mathrm{g}}$ values lower than 0.1 .

Finally, we weight the individual shear values according to their significance compared to their neighbors in the $\left(r_{\mathrm{g}}, S / N\right)$ plane. In practice, this weight factor is set to the inverse of the root mean square of the shear of the 50 nearest neighbors for a region around each galaxy size and significance. Generally, the small, faint galaxies are given a low weight and larger, bright galaxies are given a high weight because the larger galaxies are affected only by the intrinsic shape noise, while the smaller, fainter galaxies also have a significant noise component coming from sky noise in their shear measurements. In addition, subareas presenting a large shear dispersion contribute less than subareas with a low shear dispersion.

\subsection{Bias calibration}

We measure the bias of our method on the STEP2 simulations (Massey et al. 2007a) that provide images computed with various PSFs and with an added constant shear across each image. We use the sets of images characterized by a Subaru PSF with a seeing of 0.8 arcsec (PSF C). This PSF suits well our data as about half of our images are from Subaru and our image seeing lies between $0.6<\epsilon<1.0$ arcsec. However, the STEP2 images are $7 \times 7 \operatorname{arcmin}^{2}$ size, while our images are on the order of $34 \times 27 \mathrm{arcmin}^{2}$ for Suprime-Cam and $60 \times 60 \mathrm{arcmin}^{2}$ for MegaCam. Hence, the PSF should be better sampled in the true images.

Applying our reduction pipeline, we calculate the average shear of each of the 64 simulated galaxy fields and fit the difference between our shear estimate and the true shear as a function of the true shear, according to the notation of Eq. (4) from Massey et al. (2007a),

$\gamma_{i}-\gamma_{i}^{\text {true }}=m_{i} \times \gamma_{i}^{\text {true }}+c_{i}$,

where $\mathrm{i}$ is the index for both shear components. The values we found for the multiplicative biases $m_{1}$ and $m_{2}$ and the additive biases $c_{1}$ and $c_{2}$ are shown in Table 2 .

Our results compare well with those from other methods, as described in the STEP2 challenge (Massey et al. 2007a). As expected, the additive bias is rather negligible and the shear is 
slightly underestimated with the KSB method. The multiplicative bias can be seen as an evaluation of the quality of the shear measurement. Our results hence show that we can measure the galaxy shear with an accuracy better than $\sim 5 \%$. We correct each component of the shear for the multiplicative bias, and thus obtain our final shear catalog. We do not correct for the additive bias, which is strongly PSF dependent, and rather prefer to leave it as a potential systematic bias, which is small compared to the other sources of errors.

\section{Mass reconstruction}

We then translate the measured shear signal to a mass estimate. We first apply the standard Seitz \& Schneider (1995) inversion technique based on the Kaiser \& Squires (1993) algorithm to calculate a convergence density map (Sect. 4.2). This technique allows us to draw significance contour levels on the cluster image to search for structures but does not allow us to recover the true masses of objects. Indeed, the integration of the shear over a finite space introduces a constant called the mass sheet degeneracy that cannot be properly taken into account without a magnification study. To avoid this problem, we fit Navarro, Frenk, and White (NFW: Navarro et al. 1996) shear profiles on clusters to infer their 3D mass distribution in Sect. 4.3. In any case, we first have to select galaxies that lie behind the structures we aim to detect to avoid diluting the shear signal. This is described in Sect. 4.1, where we also estimate the mean background galaxy redshift, as this quantity is required to convert the shear and convergence into mass.

\subsection{Background galaxies}

\subsubsection{Color cuts}

Foreground and cluster galaxies are not lensed by the cluster. Hence, they appear as noise in the coadding of individual shear measurements and have to be deleted. The most accurate way to select background galaxies is to use spectroscopic redshifts, but it requires too much observational time. Photometric redshifts are more promising, as they are less time consuming and are starting to give accurate redshift estimations. However, we do not have spectroscopic or photometric redshifts for all galaxies and therefore we must consider galaxy colors. Galaxy colors are linked to the galaxy formation history and can be used as a crude approximation of the galaxy redshift.

We select background galaxies in a color-color diagram, comparing our galaxy colors to those from galaxy templates computed at various redshifts. We generate templates for earlyand late-type galaxies using EzGal (Mancone \& Gonzalez 2012) with Bruzual \& Charlot (2003) models, assuming a Chabrier (2003) initial mass function (IMF), a formation redshift of $z_{\text {form }}=4$, and a solar metallicity. The red early-type galaxies are modeled with a single starburst model and the blue latetype galaxies are modeled by an exponentially decaying star formation model. We remove all galaxies that correspond to the color-color area covered by template galaxies at redshift $z<z_{\text {clus }}+0.2$. For example, we show the color-color diagram of RXJ1716 with the removed area in Fig. 2. We note that the colors we use vary from one cluster to another according to the available optical bands (see Table 1). We also cut all the remaining galaxies with magnitudes brighter than $i=22$ or $r=22.5$ (depending on the image on which the shear measurement is performed), as they are very likely foreground galaxies given the high redshift of our clusters. In the same manner, galaxies fainter than $i=25$ or $r=25.5$ are removed as they are fainter than the depth of our images and, therefore, not reliable.

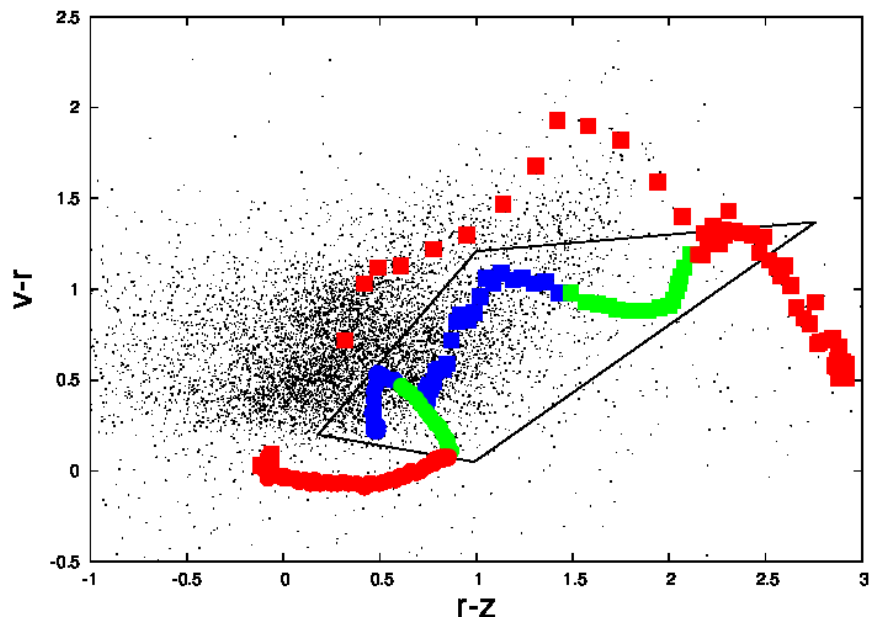

Fig. 2. $(v-r)$ versus $(r-i)$ color-color diagram for RXJ1716. Black dots represent galaxies from our catalog. Circles show late-type galaxy templates and squares early types. Green indicates templates at \pm 0.2 around the cluster redshift, blue lower redshift galaxy templates, and red higher redshift galaxy templates. The black polygon circling green and blue points corresponds to the color area we remove from our catalog. See text for details on galaxy templates used .

\subsubsection{Boost factor}

To check that the color-color cuts removed cluster dwarf galaxies, we computed the number density of galaxies in our lensing catalog as a function of radius from the brightest cluster galaxy, correcting for loss of sky area because of the presence of bright galaxies and stars in each radial bin. As a result of the magnification depletion effect (Smail et al. 1995), the number density of background galaxies should either be flat or decrease with decreasing cluster centric distance, with the exact effect depending on the slope of the change in number counts with increasing magnitude for those galaxies in, and slightly fainter, than the lensing catalog. In contrast, dwarf galaxies number density should increase with decreasing cluster centric radius, and thus any increase seen in the number density of the lensing catalog toward the cluster center is indicative that not all cluster galaxies were removed by the color cuts. The ratio of the number density of galaxies in the lensing catalog of a given annular bin compared to the number density at large cluster radius can then be used as an estimate of the contamination fraction of cluster galaxies. Under the assumption that the shapes of cluster galaxies are uncorrelated and should average to zero shear, this correction factor can then be used to boost the measured shear in the inner regions of the clusters to correct for the presence of cluster galaxies in the lensing catalog (Clowe \& Schneider 2001). It should be noted that this is a conservative estimate of the fraction of cluster galaxies as we are assuming the underlying density of background galaxies is flat and not depleted toward the cluster center. However, as the cosmic variance of the slope of the background galaxy number density with magnitude relation on arc minute sized patches can be large, estimates of the magnification depletion effect for individual clusters are too noisy to provide better constraints (Schneider et al. 2000).

We fit the radial profile of the normalized galaxy density with an exponential function of the form,

$1+f(r)=1+A \times \exp \left(-r / r_{0}\right)$

where $A$ and $r_{0}$ are constrained by the fit. We then apply this function to boost shear values in the cluster vicinity. The weights 


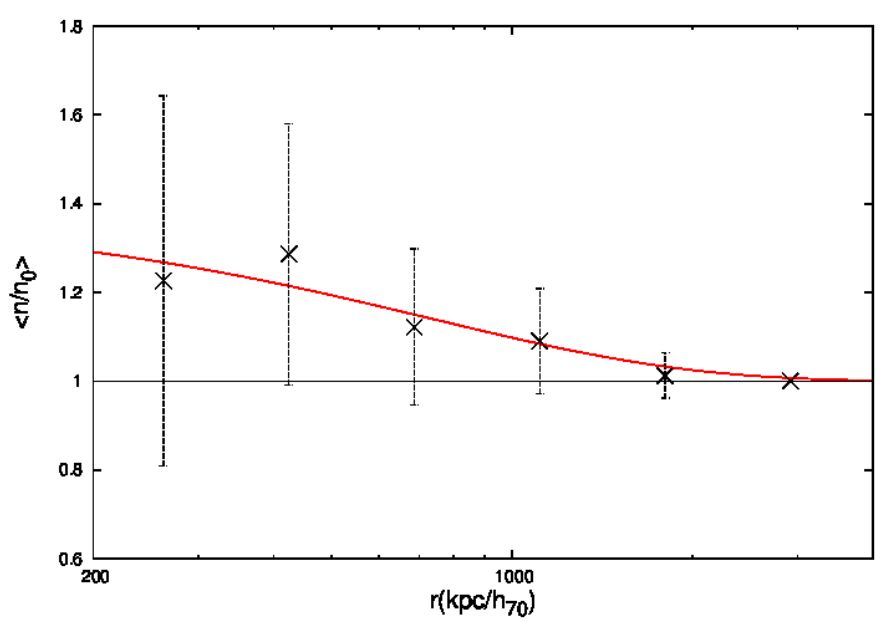

Fig. 3. Stacked normalized galaxy density profile for all clusters. Error bars are the dispersion of values in the stack. Radius is in comoving distance and in kpc units. Individual profiles are centered on the WL peak. The red curve is the best exponential fit (see Eq. (5)) to the data.

are also modified according to the error on the fit to the density profile. We show the stacked normalized galaxy density along with the best fit for our boost factor in Fig. 3. The error bars are computed from the dispersion over all clusters and show that the boost factor varies from one cluster to another, requiring individual fits. The galaxy density profiles are computed using the WL peak as the center. As a sanity check, we also computed the density profiles centered on the brightest cluster galaxy (BCG) and found no significant variation in the mass estimates of our clusters. We neglected the effect of magnification when estimating the radial galaxy density profile, but Okabe \& Smith (2015) showed that this only decreases the amplitude of the shear profile by $\sim 10 \%$ on scales lower than one-tenth of the virial radius. Applying the corrections above as a function of radius from the cluster center results in an increase in the measured cluster masses. To be exact, the boost factor affects the concentration and then the mass as we fixed the concentration parameter to break the mass concentration degeneracy (see Sect. 4.3). The largest increase in mass is $30 \%$ (MACJ0717), while the mean increase is $9 \%$ and the median $6 \%$.

\subsubsection{Distance measurements}

Another issue is to measure the distances of the lens and of the background galaxies. These observables are required to estimate the mass of the lens, which depends on the ratio of the sourceto-observer distance over the source-to-lens distance: $D_{\mathrm{s}} / D_{\mathrm{ls}}$. We estimate the lens distance through the spectroscopic redshift of the cluster. The classical way of estimating the mean background galaxy distance is to average the distance ratio $D_{\mathrm{s}} / D_{\mathrm{ls}}$ over all source galaxies; see also Applegate et al. (2014) for a method that uses all galaxy background photometric redshifts in a Bayesian formalism.

As we do not have photometric redshifts for background galaxies, we consider an external redshift distribution. We use the COSMOS data (Ilbert et al. 2009) as our redshift distribution. These data are suitable as they cover a large area of about $1.7 \mathrm{deg}^{2}$ after masking, down to a magnitude of $i=25$, and are adapted to our redshift range. Furthermore, the photometric redshifts of COSMOS are computed with a high precision, using 30 bands from near-UV to mid-IR. We first apply the same magnitude and color cuts as those applied to our shear catalog. We then remove all galaxies that have a photometric redshift that is smaller than that of the cluster, and we calculate the mean of the ratio of the source to lens versus source distances $D_{\mathrm{ls}} / D_{\mathrm{s}}$, applying an appropriate weight. The weighting function is generated on the COSMOS galaxy subsample from a $2 \mathrm{D}$ polynomial fitted on the shear weighting function in our data in a half-light radius versus magnitude plane. We use the magnitude instead of the $\mathrm{S} / \mathrm{N}$ ratio as the second coordinate because the $\mathrm{S} / \mathrm{N}$ in COSMOS and in our data can vary significantly. Finally, the weights generated on COSMOS are renormalized to 1 . The mean redshift of background galaxies is then set to the redshift that allows us to find the measured mean distance ratio $D_{\mathrm{ls}} / D_{\mathrm{s}}$. These redshifts can be found in Table 3 .

\subsection{Two-dimensional mass map}

We reconstruct the projected convergence field by inverting the shear in Fourier space, following Seitz \& Schneider (1995). This technique is an iterative application of the Kaiser \& Squires (1993) algorithm to correct for the fact that we measure the reduced shear, which is equal to the shear $\gamma$ divided by $1-\kappa$, and not the shear. We reconstruct the first convergence map assuming $\kappa=0$ in the shear, and then generate a map from the shear where the convergence is set to the previous map in the loop until the process converges. We find that the convergence map remains constant within $0.01 \%$ after three realizations. This technique allows us to better estimate the mass map around high masses and is therefore particularly suitable for our cluster mass reconstruction. The convergence field is smoothed with a Gaussian filter of width $\theta_{\mathrm{s}}=1$ arcmin at each step of the algorithm, before reading off which convergence to use to correct for a given galaxy. The noise level in the final convergence map can be estimated as Eq. (6) (van Waerbeke 2000),

$\sigma_{\kappa}=\frac{\sigma_{\epsilon}}{\sqrt{4 \pi n_{\mathrm{bg}} \theta_{\mathrm{s}}^{2}}}$,

where $n_{\mathrm{bg}}$ is the density of background galaxies and $\sigma_{\epsilon}$ the dispersion of the ellipticities of the background galaxies. The values $n_{\mathrm{bg}}$ and $\sigma_{\epsilon}$ are estimated independently for each image, taking the weight function of the shear into account. The parameter $\sigma_{\epsilon}$ ranges from 0.27 to 0.32 across our data, while $n_{\text {bg }}$ can be found in Table 3 for each cluster.

One can then convert the convergence map into a surface mass density map using the following definition of the convergence (Eq. (7)):

$\kappa=\frac{\Sigma}{\Sigma_{\text {crit }}}$

where $\Sigma$ is the surface mass density and $\Sigma_{\text {crit }}$ is the critical surface mass density defined in Eq. (8), i.e.,

$\Sigma_{\text {crit }}=\frac{c^{2}}{4 \pi G} \frac{D_{\mathrm{s}}}{D_{\mathrm{l}} D_{\mathrm{ls}}}$

$c$ is the speed of light, $G$ the gravitational constant, and $D_{\mathrm{s}}, D_{\mathrm{l}}$, and $D_{\mathrm{ls}}$ are the distance to the source, distance to the lens, and distance between the source and the lens, respectively. This conversion hence only requires the knowledge of the lens and source redshifts, which is calculated in Sect. 4.1. As we cannot properly account for the mass sheet degeneracy in our reconstruction, we did not try to estimate the mass of clusters through the convergence map. These mass maps are thus only used to detect clusters and their surrounding structures, while the cluster masses 

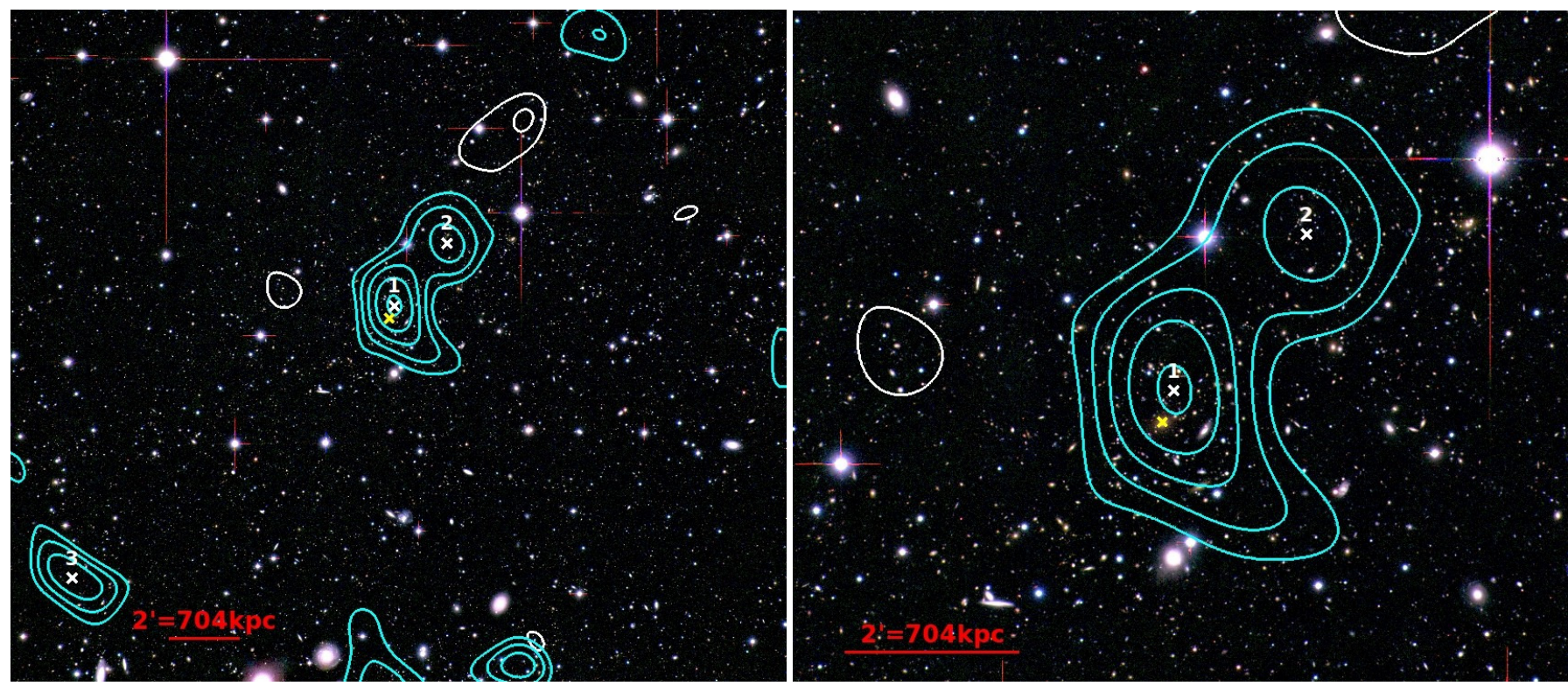

Fig. 4. Convergence density map for MACSJ1621 overlaid on three-color image. Contour levels (cyan) are shown in signal-to-noise from $3 \sigma_{\kappa}$ and by step of $1 \sigma_{\kappa}$. The white contours show the convergence density map computed from the rotated shear with the same signal-to-noise levels. We note that the signal corresponding to the cluster disappears in this reconstruction. Weak lensing peaks are indicated with a white cross starting with the highest detection. The yellow cross indicates the position of the BCG. The left panel shows the full image and the right panel shows a zoom on the cluster region.

are estimated in the next section fitting an NFW profile to the shear.

The significance of the detection is computed from a noise resampling technique, adding a random ellipticity to every galaxy for each realization. To preserve the shape noise properties of the sample, we draw the added ellipticities from the image galaxy catalog. With this approach, we neglect the additional shear signal, as it is very unlikely that it correlates with the detected structures given the large number of galaxies in our catalogs. The shape noise used in Eq. (6) is increased by a factor of $\sqrt{2}$ as the ellipticity of galaxies now corresponds to the sum of two Gaussian distributions with a null mean and a width $\sigma_{\epsilon}$. We perform 100 realizations for each catalog, computing the detection level of every structure at each step. The mean and dispersion of these detection levels give a strong estimate of the significance of the detection. We also measure the number of realizations in which the structure is detected at more than $3 \sigma$ above the map noise. For example, we can be very confident in a structure detected at more than $3 \sigma$ in $95 \%$ of the realizations. In addition, this noise resampling allows us to refine the measure of the position of each structure, computing the mean and dispersion of the local maximum position over all noise realizations. These quantities correspond to an estimate of the structure center and to the error on its position, respectively .

For example, in Fig. 4 we show the three-band color image with the convergence contours overlaid for MACSJ1621. The contours are spaced in units of the map noise computed from Eq. (6), starting at $3 \sigma$. We show the same figure for every cluster with X-ray emissivity and galaxy light density contours when available in Sect. 6. As a sanity check, we computed the mass map with shear rotated by 45 degrees (white contours) and found that the signal due to the cluster presence disappears in this map, validating our convergence map reconstruction method. The position of the WL peaks are indicated with white crosses with a 1 for the cluster and a 2 for the main secondary structure. The cluster is detected at $(6.8 \pm 1.4) \sigma_{K}$ in the center region and an elongated structure aligned with the cluster major axis can be seen at a $(5.9 \pm 1.7) \sigma_{\kappa}$ confidence level computed from the mean and dispersion of 100 realizations of the noise. These two structures are detected in $97 \%$ and $96 \%$ of the realizations, respectively. The nature of the secondary peak is discussed in Sect. 6 comparing the WL with other probes (X-ray and optics). The center positions are estimated with a precision of about $200 \mathrm{kpc}$. Also, we note an offset between the BCG denoted by a yellow cross and the WL peak. This offset is discussed in Sect. 5.3, in which we estimate cluster masses at both positions.

In spite of all our care to build accurate mass maps, some peaks arise from the noise. One must evaluate the number of these fake peaks to discuss the detection of structures in the mass maps. As the number of fake peaks depends both on the density of background galaxies and on the redshifts of the lens and sources, we compute the fake peak probability for each cluster field. To accomplish this, we assign a random position to each galaxy in the frame to make sure that no structure from the original positions would be left in the simulation. We then use this new ellipticity catalog as an input to our mass map pipeline. The resulting convergence map should be representative of the noise. However, the presence of the cluster also modifies the distribution of fake peaks. To take this into account, we add shear values based on the fitted NFW profile of the corresponding cluster to the ellipticity of each galaxy (see Sect. 4.3). We find slightly fewer peaks when adding the cluster. This is because some noise peaks can be aligned with the cluster, and also because the presence of the cluster is compensated by negative convergence values in the mass map as the mean convergence in the reconstruction is set to zero. We carry out 100 realizations to capture the statistical properties of the fake peaks. For MACSJ0717, we also perform 10000 realizations to check that our 100 realizations are sufficient. We find little difference between the two cases. Quantitatively, we find 11.1 peaks above $3 \sigma_{\kappa}$ and 1.3 above $4 \sigma_{\kappa}$ in the entire Suprime-Cam field for 100 realizations, and 10.9 and 1.2 above $3 \sigma_{\kappa}$ and $4 \sigma_{\kappa}$ for 10000 realizations. In any case, we find less than 0.1 fake peaks above $5 \sigma_{\kappa}$. When discussing the detection of structures in Sect. 6, we give the expected number 

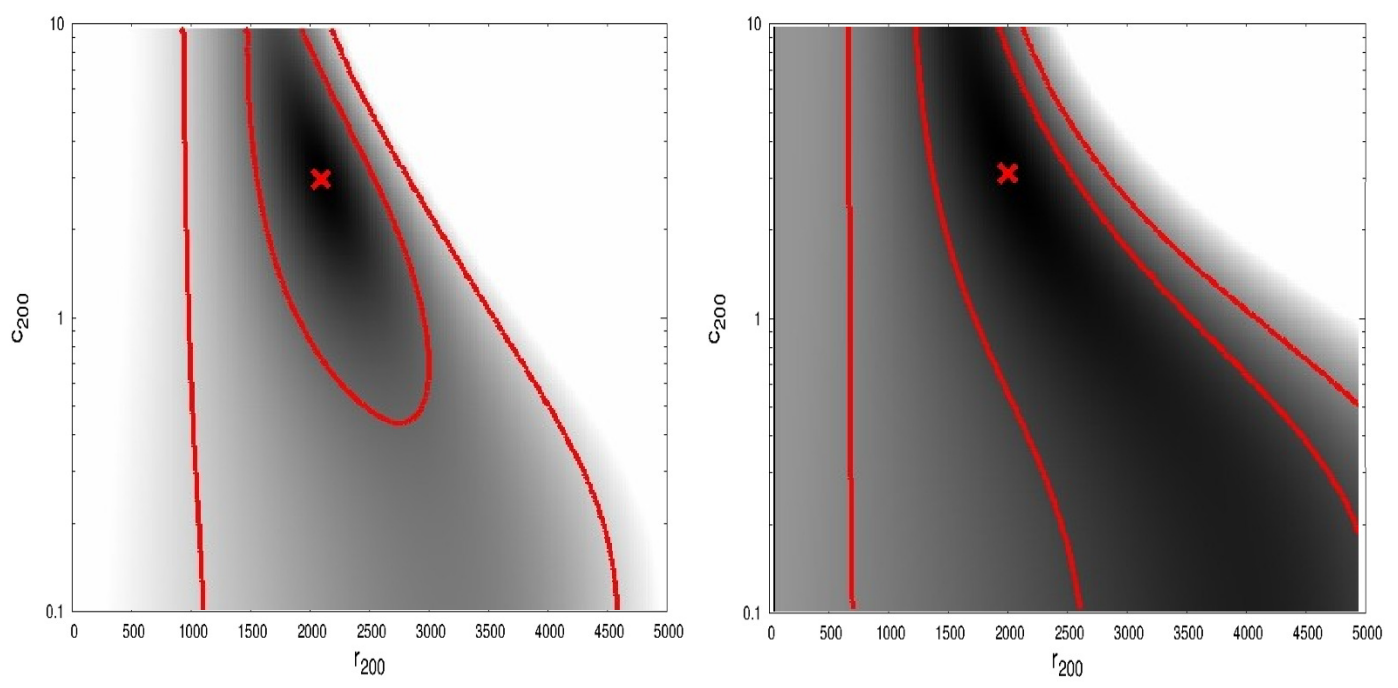

Fig. 5. Degeneracy between the $r_{200}$ and $c_{200}$ NFW profile parameters for the best fit of MACSJ0717 (left, high significance) and NEP200 (right, low significance). The shaded region represents the $\Delta \chi^{2}$, with 1 and $2 \sigma$ contours in red. The red cross indicates the best-fit value.

of fake peaks in the displayed area for each cluster. We note that in Fig. 4, the white contours corresponding to the reconstruction of the orthonormal shear component are in good agreement with the expected number of fake peaks for the field shown (2.9 above $3 \sigma_{\kappa}$ and 0.4 above $4 \sigma_{\kappa}$ in the left-hand field).

\subsection{Cluster mass fit}

To infer the cluster mass distribution, we choose to fit the shear profile centered on the cluster. This avoids having to measure the shear in the cluster core, and partially breaks the mass sheet degeneracy by imposing a given mass profile on the data. We note that using this radial technique on $N$-body simulated clusters, Becker \& Kravtsov (2011) and Bahé et al. (2012) found a systematic underestimate of cluster masses of roughly $5 \%$, which we do not correct for as the exact correction factor is likely to be a function of the chosen cosmologcial paramaters (and is small compared to the uncertainties for all of our clusters). The NFW density profile (Navarro et al. 1996) defined in Eq. (9) is among the best available profiles to fit observed galaxy clusters (e.g., Umetsu et al. 2011). This profile is written as

$\rho_{\mathrm{NFW}}(r)=\frac{\rho_{\mathrm{s}}}{\frac{r}{r_{\mathrm{s}}}\left(1+\frac{r}{r_{\mathrm{s}}}\right)^{2}}$

where $r_{\mathrm{s}}$ is the scale radius and $\rho_{\mathrm{s}}$ a density expressed as $\rho_{\text {crit }} \delta_{\mathrm{c}}$. $\rho_{\text {crit }}=3 H^{2} / 8 \pi G$ is the critical density of the Universe at the cluster redshift, and $\delta_{\mathrm{c}}$ is a dimensionless density that depends on the DM halo, and that can be expressed as a function of the concentration parameter,

$\delta_{\mathrm{c}}=\frac{\Delta}{3} \frac{c_{\Delta}^{3}}{\ln \left(1+c_{\Delta}\right)-\frac{c_{\Delta}}{1+c_{\Delta}}}$,

where $\Delta$ is the overdensity compared to the critical density and $c_{\Delta}=r_{\Delta} / r_{\mathrm{s}}$ is the concentration parameter. By integration of the density under spherical symmetry, the mass $M_{\mathrm{NFW}, \Delta}$ in a given radius $r_{\Delta}$, can be estimated as a function of $r_{\Delta}$ and $c_{\Delta}$ only

$M_{\mathrm{NFW}, \Delta}=\frac{4 \pi \rho_{\mathrm{s}} r_{\Delta}^{3}}{c_{\Delta}^{3}}\left[\ln \left(1+c_{\Delta}\right)-\frac{c_{\Delta}}{1+c_{\Delta}}\right]$.

The radial shear profile has an analytic formula derived in, for example, Wright \& Brainerd (2000). We fit this formula to the measured shear to obtain $r_{\Delta}$ and $c_{\Delta}$, which are converted into a cluster mass according to Eq. (11). There is a known degeneracy between the concentration $c_{\Delta}$ and the mass $M_{\Delta}$ (e.g., Diemer \& Kravtsov 2014; Meneghetti et al. 2014), or equivalently $r_{\Delta}$ in our case. We show in Fig. 5 the degeneracy between both parameters of our NFW fit for two clusters representative of the large (MACSJ0717) and low (NEP200) significance detections. These plots highlight the need to break the degeneracy between the two parameters especially in the low significance case. This can be achieved using predictions of the typical concentration of clusters from cosmological $N$-body simulations, and one can either choose a mean concentration for all clusters in the sample (e.g. Applegate et al. 2014) or use a mass-redshift-concentration relation (e.g., Hoekstra et al. 2015). To break the degeneracy between $r_{\Delta}$ and $c_{\Delta}$, we fix the concentration parameter to $c_{200}=3.5$, since Gao et al. (2008) demonstrated that very massive clusters have concentration parameters between 3 and 4 at the studied redshifts. This choice of a fixed concentration parameter imposes a systematic error on each individual cluster mass although the average should be correct. We quantify the error on the mass measurement due to the intrinsic scatter of 1.34 on the concentration parameter estimate in Gao et al. (2008) by fixing the concentration parameter to 2.16 and 4.84, which represent the scatter around our chosen value of $c_{200}=3.5$. We find a variation of the mass of about $\pm 25 \%$. This error is not added to the error budget of Table 3 . As a result of our choice of breaking the mass-concentration degeneracy by fixing the concentration parameters, any concentration effect, such as the boost factor (see Sect. 4.1.2) or the off-centering effect (see Sect. 5.3), directly affects the mass estimate.

The fit is performed in an annulus where the inner radius is iteratively set to a value larger than the Einstein radius, to remove the area affected by strong lensing. We also require there be a minimum number of objects in every bin, which can push the inner radius to large physical values in the case of high redshift clusters. The outer radius is set to the value at which the output $r_{\Delta}$ does not significantly change (less than $1 \%$ ) if we probe a larger area. We also ensure that the outer radius is at least larger than the output $r_{\Delta}$. The fit is performed on the tangential shear computed to the cluster center, which is defined as the highest peak close to the cluster position in the convergence map reconstruction. We discuss the possibility of using the BCG instead 
Table 3. Results on galaxy clusters.

\begin{tabular}{|c|c|c|c|c|c|c|c|c|}
\hline Cluster & $z$ & $\bar{z}_{\mathrm{bg}}$ & $\begin{array}{c}n_{\mathrm{bg}} \\
\left(\operatorname{arcmin}^{-2}\right)\end{array}$ & $\begin{array}{c}r_{200}^{\mathrm{NFW}} \\
\left(\mathrm{kpc} h_{70}^{-1}\right)\end{array}$ & $\sigma_{\mathrm{NFW}} / \sigma_{2 D}$ & $\begin{array}{c}M_{200}^{\mathrm{NFW}} \\
\left(10^{14} M_{\odot} h_{70}^{-1}\right)\end{array}$ & $\begin{array}{c}M_{500}^{\mathrm{NFW}} \\
\left(10^{14} M_{\odot} h_{70}^{-1}\right)\end{array}$ & $\begin{array}{c}M_{500}^{\mathrm{X}} \\
\left(10^{14} M_{\odot} h_{70}^{-1}\right)\end{array}$ \\
\hline XDCS0329 & 0.4122 & 0.90 & 10.20 & - & $1.2 / 2.8$ & - & - & $2.9 \pm 0.6$ \\
\hline MACSJ0454 & 0.5377 & 0.99 & 9.96 & - & $1.9 / 5.1$ & - & - & $13.9 \pm 3.0$ \\
\hline ABELL0851 & 0.4069 & 0.92 & 8.30 & $1542 \pm 160$ & $3.9 / 7.6$ & $6.6 \pm 2.0$ & $4.4 \pm 1.4$ & $5.5 \pm 1.2$ \\
\hline LCDCS0829 & 0.4510 & 0.93 & 8.79 & $1638 \pm 218$ & $3.8 / 5.5$ & $8.5 \pm 3.2$ & $5.7 \pm 2.1$ & $16.9 \pm 3.6$ \\
\hline MS1621 & 0.4260 & 0.93 & 14.13 & $1718 \pm 140$ & $6.4 / 8.3$ & $9.2 \pm 2.2$ & $6.2 \pm 1.5$ & $4.5 \pm 0.5^{M 12}$ \\
\hline $\mathrm{OC} 02$ & 0.4530 & 0.96 & 13.15 & $1202 \pm 187$ & $3.1 / 4.7$ & $3.4 \pm 1.5$ & $2.3 \pm 1.0$ & - \\
\hline NEP200 & 0.6909 & 1.02 & 5.80 & $1929 \pm 306$ & $3.3 / 5.1$ & $18.9 \pm 8.2$ & $12.7 \pm 5.5$ & - \\
\hline RXJ2328 & 0.4970 & 0.95 & 11.46 & $1393 \pm 159$ & $3.2 / 5.5$ & $5.5 \pm 1.9$ & $3.7 \pm 1.2$ & $2.2 \pm 0.5$ \\
\hline CLJ0152 & 0.8310 & 1.19 & 14.94 & $1670 \pm 194$ & $3.8 / 8.3$ & $14.0 \pm 4.6$ & $9.4 \pm 3.1$ & $8.8 \pm 1.9$ \\
\hline MACSJ0717 & 0.5458 & 0.98 & 13.16 & $2236 \pm 206$ & $5.2 / 10.9$ & $23.6 \pm 6.4$ & $15.9 \pm 4.3$ & $17.8 \pm 1.7^{M 12}$ \\
\hline BMW1226 & 0.8900 & 1.43 & 10.12 & - & $0.2 /-$ & - & - & $12.1 \pm 0.4$ \\
\hline MACSJ1423 & 0.5450 & 0.93 & 8.98 & $1594 \pm 214$ & $3.4 / 5.0$ & $8.8 \pm 3.3$ & $5.9 \pm 2.2$ & $5.7 \pm 1.2$ \\
\hline MACSJ1621 & 0.4650 & 0.94 & 16.39 & $1379 \pm 185$ & $4.2 / 6.8$ & $5.2 \pm 1.9$ & $3.5 \pm 1.3$ & $4.3 \pm 0.4^{M 12}$ \\
\hline RXJ1716 & 0.8130 & 1.17 & 7.49 & $1685 \pm 194$ & $3.9 / 7.3$ & $14.1 \pm 4.7$ & $9.5 \pm 3.2$ & $2.8 \pm 0.5^{M 12}$ \\
\hline MS2053* & 0.5830 & 0.98 & 14.44 & $1620 \pm 195$ & $4.6 / 8.7$ & $9.5 \pm 3.3$ & $6.4 \pm 2.2$ & $4.9 \pm 1.1$ \\
\hline CXOSEXSI2056* & 0.6002 & 0.98 & 14.44 & - & $0.7 / 4.4$ & - & - & $3.6 \pm 0.8$ \\
\hline
\end{tabular}

Notes. The first eight clusters are observed with CFHT/Megacam and the last eight with Subaru/Suprime-cam. The different columns correspond to \#1: cluster ID; \#2: cluster redshift; \#3: mean redshift of background galaxies; \#4: mean galaxy density of the background galaxies; \#5: $r_{200}$ from the best NFW fit; \#6: significance of the NFW fit/significance of the WL peak in the 2D mass map; \#7: $M_{200}^{\mathrm{NFW}}$ from the best NFW fit centered on the WL peak; \#8: $M_{500}^{\mathrm{NFW}}$ computed in $r_{500}$ from $M_{200}^{\mathrm{NFW}}$, assuming the same NFW profile; and \#9: total masses in $r_{500}$ derived from XMM X-ray data from Guennou et al. (2014) or Chandra X-ray data from the Maughan et al. (2012) sample denoted by the symbol ${ }^{M 12}$ and computed in Laganá et al. (2013). ${ }^{(*)}$ CXOSEXSI_J205617 and MS_2053.7-0449 are on the same image.

of the WL peak as the center in Sect. 5.3, but we mainly discuss masses centered on the WL peak in the following. Cluster masses are shown in Table 3.

An estimate of the significance of the fit is obtained by computing the $\Delta \chi^{2}$ between the best-fit NFW model and a zero mass model. The tangential shear profiles for every cluster can be found in Appendix B, where the error bars correspond to the orthonormal shear that should be equal to zero in the absence of noise. We measure $r_{200}$ from the best NFW fit and then compute $M_{200}$, and $M_{500}$. We note that for clusters where the NFW fit has a low significance value $(\sigma<3)$, the tangential shear profiles present error bars that are consistent with no signal. We then do not compute a mass for these clusters, as their shear profiles are not reliable.

The errors are computed using the same noise resampling method as for the mass maps (see Sect. 4.2). A random ellipticity is drawn from our catalog and added to each galaxy. Then, the best NFW fit gives a new value for $r_{200}$ and $M_{200}$. The mean and dispersion over 100 noise realizations are used as the true value and its error. The $r_{200}$ and various mass values are given in Table 3 of Sect. 5.

\section{Galaxy clusters}

In this section we present the results concerning the 16 galaxy clusters that we have studied. The discussion is based on the masses obtained from the NFW fits presented in Sect. 4.3 and given in Table 3. After discussing the WL masses (Sect. 5.1), we compare them to the X-ray values from the literature (Sect. 5.2), and then analyze the effect of using the BCG as the cluster center instead of the WL center (Sect. 5.3). We compare individual cluster masses with other studies jointly with the environment discussion in the next section (Sect. 6).

\subsection{Weak lensing masses}

The results of the best NFW fit are given only when its significance is higher than $3 \sigma$, because otherwise such a mass would not be reliable. This means that we were not able to constrain the masses of all clusters (see Table 3 and shear profiles in Appendix B). The fact that some of our fits do not converge can have several explanations depending on each case. One obvious limitation is the background galaxy density; as the noise is proportional to the inverse square root of the background density, the deeper the observations, the higher the signal-to-noise of the shear. The data obtained with Subaru, which is an 8-m class telescope, are less affected than those obtained with the CFHT, which is only a 4-m class telescope. The masses of the clusters and the noise in the images are also important factors. A high mass cluster tends to be detected even with a low background galaxy density. Finally, we note that the redshift of the cluster also plays a role. For example, BMW-HRI J122657 is a rather massive cluster, but at a redshift of $z=0.89$. As the lensing effect is measured on the galaxies behind the cluster, the higher the redshift, the more difficult it is to detect the cluster. A redshift of $z \sim 0.9$ is close to the accessible limit, as lensing is most sensitive to structures at redshifts around $z \sim 0.3-0.4$.

We present the individual shear profiles in Appendix B. In Fig. 6 we show a stacked shear profile including all 12 clusters for which it was possible to compute a mass. The black dots correpond to the stacking of all individual cluster shear profiles, and the error bars are the dispersion of each shear bin values. In addition we also coadd the shear catalogs recentered to the WL peak and compute a global shear profile, using the mean redshift of the clusters to convert into comoving distance (blue points). In this case the error bars correspond to the rotated shear as for the individual profiles. Both methods agree very well. In the second case, the error bars are smaller because we get more galaxies per radial bin, but this does not take into account the dispersion in 


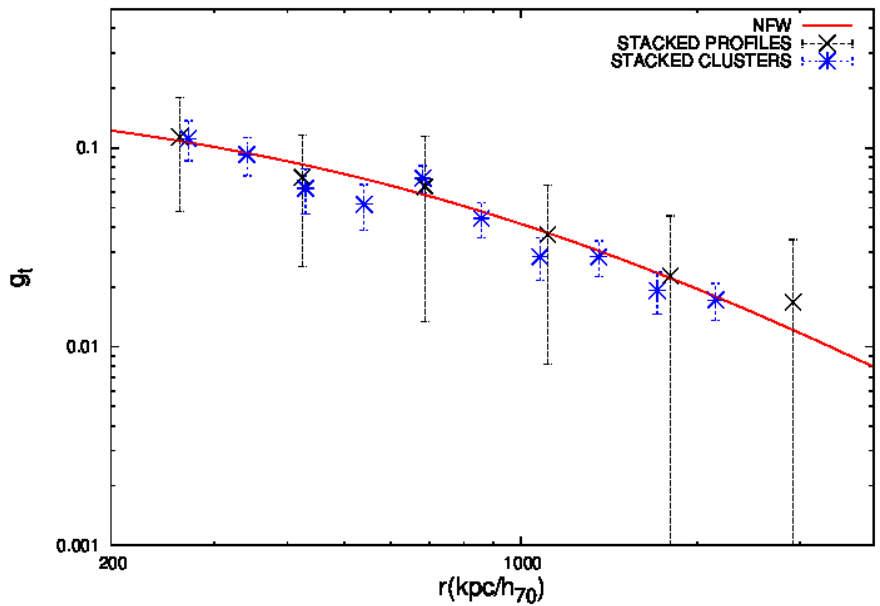

Fig. 6. Stacked shear profile for the 12 clusters for which we were able to safely measure the mass. Black points correspond to the stacked profiles and blue points to the profile of the stacked shear catalogs (see text for details). In the first case error bars are the dispersion of values in the stack, and in the second case the rotated shear. Radius is in comoving distance and in kpc units. Individual profiles are centered on the WL peak. The red curve is the best NFW fit to the stacked profile.

the shears. In our study, we have enough signal-to-noise in each cluster to stack the individual shear profiles as well. Though the error bars are still large given that we have only a small number of clusters, most of the noisy or asymmetrical irregularities have been washed out and the stacked shear profile is well represented by an NFW spherical profile.

For the clusters for which we were able to compute masses, we find error bars typical of WL studies. We note, however, that using the noise resampling method to determine the mass increases our errors over using only the significance of the best NFW fit. We choose to show the former errors because they are more robust and more conservative. We do not statistically compare our masses with other WL studies because we have only few clusters in common. Three of our clusters are studied in the Mahdavi et al. (2013) sample, three in the CCCP sample (Hoekstra et al. 2015), three in the Weighting the Giants sample (Applegate et al. 2014), two in the Foëx et al. (2012) sample, one is studied in Jauzac et al. (2012) and Medezinski et al. (2013), and one in Israel et al. (2014). Nonetheless, we compare the WL masses, and the X-ray and strong lensing estimates for each cluster in Sect. 6.1. In the next subsection, we compare our WL masses with those derived from X-rays to evaluate potential biases in both measurements.

\subsection{X-ray and WL masses}

The X-ray masses come from two different samples. Most of them have XMM-Newton data and are taken from Guennou et al. (2014). We add four clusters that have Chandra data and belong to the Maughan et al. (2012) sample. MACSJ1423 has Chandra data but is also part of Guennou et al. (2014). The masses from Guennou et al. (2014) are obtained by applying the Kravtsov et al. (2006) scaling relation to the X-ray derived temperature of the clusters. The error bars were recomputed taking the scatter of this scaling relation into account, since they were too optimistic in Guennou et al. (2014). The masses from Chandra observations were computed in Laganá et al. (2013) using both the temperatures and surface brightness profiles (see Eq. (5) of the mentioned paper).

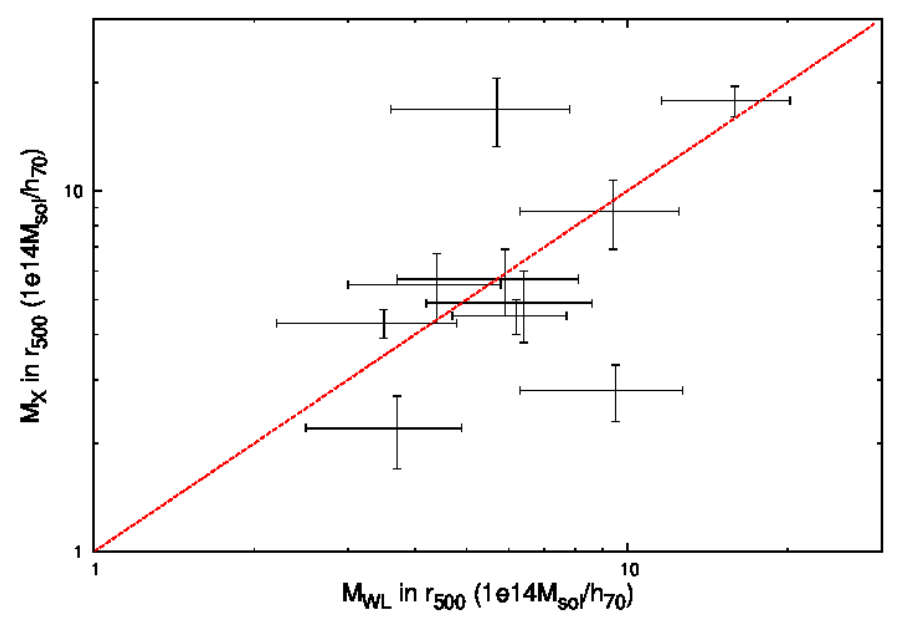

Fig. 7. X-ray versus WL masses. The red dashed line is the first bisector and represents the sequence on which X-ray and WL masses would be equal. All values can be found in Table 3 .

In Fig. 7 we compare the cluster masses inferred from X-ray data and from WL, which are all computed in $r_{500}$ for the ten clusters that have both data. We see that the points are fairly distributed around the line of equality. Computing the lognormal mean ratio of the WL to X-ray masses, we find that WL masses are $8 \%$ higher than the X-ray masses in the mean. Finding an offset is normal, as the X-ray masses rely on the assumption that clusters are relaxed, which is generally not the case. Weak lensing, on the other hand, does not need such an assumption, and WL masses are usually more reliable. An underestimate of about 10 to $40 \%$ in the X-ray derived total cluster masses is commonly observed (Rasia et al. 2006; Nagai et al. 2007; Battaglia et al. 2013). We also note a departure from this relation for LCDCS0829, for which we cannot reproduce the high X-ray mass, and for RXJ1716, which has a very low mass in X-rays compared to its WL mass. In the first case, we note that LCDCS0829 is highly asymmetrical as seen from its mass map in Fig. 12 (Sect. 6). Hence, the hypothesis of spherical symmetry that we made for our NFW fit might explain why we find a low mass for this cluster. In general one can expect WL masses to be very accurate for individual clusters, but only for a large sample of clusters.

\subsection{Brightest cluster galaxy and weak lensing offset}

In this section, we discuss the difference in the mass estimate when centering on the BCG instead of the WL peak. We chose the latter center and apart from this section our WL masses discussed in this paper are computed centered on the WL peak.

First, using our simulated clusters (see Sect. 4.2) for different realizations of the noise, we measure the offset between the true input center and the highest WL peak. We find a mean offset of 0.32 arcmin with a scatter of 0.20 arcmin. We use angular distances here because the noise comes from the background galaxies. We can then say that using the BCG as the center of mass of the cluster is a good approximation only if the offset of the BCG and WL peak is lower than 0.52 arcmin (one sigma above the mean offset owing to the noise). For each realization, we also compute the mass centered on the true input center and on the highest WL peak. We find that centering on the WL peak systematically overestimates masses by about $8 \%$ in the mean with a scatter of $9 \%$. 


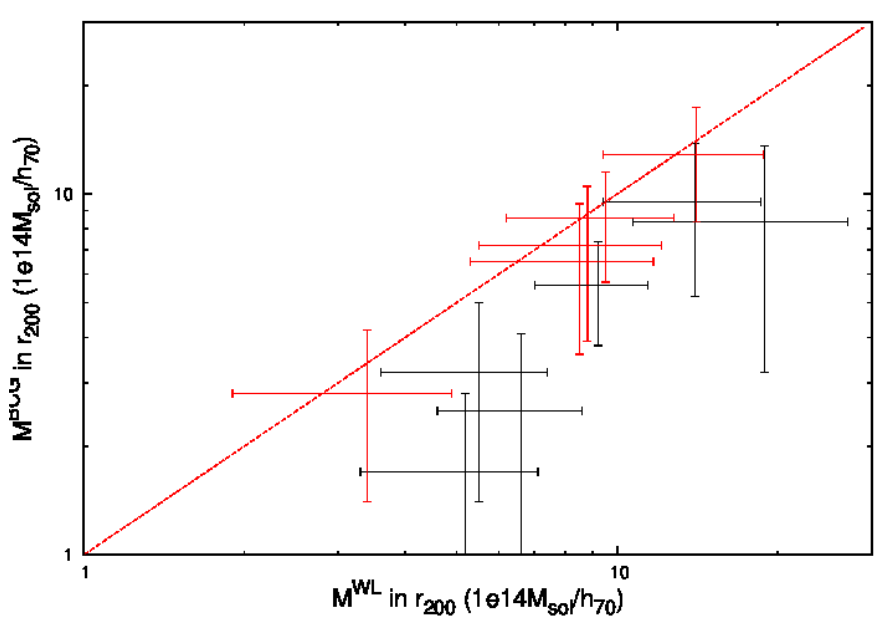

Fig. 8. WL masses centered on the BCG versus WL masses centered on the WL peak. Red dots correspond to clusters for which the WL peak is closer than 0.52 arcmin from the BCG, and black dots for those with higher position offsets. The red dashed line is the first bisector and represents the sequence on which both masses would be equal. The different values can be found in Table 4. We note that NEP200 lies in the large offset category, even if its offset is slightly lower than 0.52 arcmin, because of its mass map reconstruction (see text for details).

For clusters that have a well-identified BCG, we then compute the WL masses centered on the BCG in our data. The resulting masses are shown in Table 4 . We also plot one mass estimate against the other in Fig. 8. Table 4 provides the offset between the BCG and the WL peak, which can be high for some clusters. The mean angular distance between the WL and BCG centers is 0.67 arcmin and ranges from 0.29 arcmin to 1.20 arcmin. We also show the BCG offset in comoving distance in Table 4 to allow a comparison with the shear profiles that are computed within comoving radii. The mean offset between the BCG and DM centers in comoving distance is $246 h_{70}^{-1} \mathrm{kpc}$, which is about $100 \mathrm{kpc}$ higher than what is observed at lower redshift (e.g., Oguri et al. 2010), and highlights the fact that our clusters are mostly not relaxed and have probably suffered from a complex merging history. According to our simulations, we can distinguish between two populations of clusters. Those with a BCG offset that is lower than 0.52 arcmin and those with a larger offset. For the first category, the BCG offset is compatible with the noise offset. Thus the BCG center assumption is valid and the masses centered on the BCG and WL peak should agree. In the second case, the BCG is likely not the center of mass of the cluster, and masses centered on the BCG and on the WL peak significantly disagree. Additionally, we verify that clusters with small BCG offsets are indeed not ongoing mergers, looking at their convergence map. Only NEP200 presents signs of an ongoing merger with two peaks in the WL reconstruction, and is then counted in the merger category. We also note that the BCG offset for this cluster is very close to the acceptable limit. These expectations are met well in Fig. 8, where we isolated the two types of clusters. When identifying clusters that have their BCG and WL peaks closer than 0.52 arcmin (red dots), we find that masses with the different centers agree well within the error bars. However, the error bars are not independent for the two measurements as the shear at large radius are largely the same. The WL masses are still slightly higher when centered on the WL peak because centering on the WL peak maximizes the positive contribution of noise to the mass. Hence, choosing the center the way we did tends to overestimate the mass in relaxed clusters compared to centering on the BCG. The masses are lower by
Table 4. Comparison of masses centered on the WL peak and on the BCG for 11 clusters.

\begin{tabular}{lcccc}
\hline \hline Cluster & $\begin{array}{c}d_{|\mathrm{WL}-\mathrm{BCG}|}^{\text {com }} \\
\left(\mathrm{kpc} h_{70}^{-1}\right)\end{array}$ & $\begin{array}{c}\theta_{|\mathrm{WL}-\mathrm{BCG}|} \\
(\operatorname{arcmin})\end{array}$ & $\begin{array}{c}M_{200}^{\mathrm{NFW}} \\
\left(10^{14} M_{\odot} h_{70}^{-1}\right)\end{array}$ & $\begin{array}{c}M_{200}^{\mathrm{NFW}, \mathrm{BCG}} \\
\left(10^{14} M_{\odot} h_{70}^{-1}\right)\end{array}$ \\
\hline ABELL0851 & 384 & 1.18 & $6.6 \pm 2.0$ & $2.5 \pm 1.6$ \\
LCDCS0829 & 178 & 0.51 & $8.5 \pm 3.2$ & $6.5 \pm 2.9$ \\
MS1621 & 338 & 1.01 & $9.2 \pm 2.2$ & $5.6 \pm 1.8$ \\
OC02 & 74 & 0.21 & $3.4 \pm 1.5$ & $2.8 \pm 1.4$ \\
NEP200 & 209 & 0.49 & $18.9 \pm 8.2$ & $8.4 \pm 5.2$ \\
RXJ2328 & 343 & 0.94 & $5.5 \pm 1.9$ & $3.2 \pm 1.8$ \\
CLJ0152 & 339 & 0.74 & $14.0 \pm 4.6$ & $9.5 \pm 4.3$ \\
MACSJ1423 & 146 & 0.38 & $8.8 \pm 3.3$ & $7.2 \pm 3.3$ \\
MACSJ1621 & 422 & 1.20 & $5.2 \pm 1.9$ & $1.7 \pm 1.1$ \\
RXJ1716 & 190 & 0.42 & $14.1 \pm 4.7$ & $12.9 \pm 4.5$ \\
MS2053 & 87 & 0.29 & $9.5 \pm 3.3$ & $8.6 \pm 2.9$ \\
\hline
\end{tabular}

Notes. The first six clusters are observed with CFHT/Megacam and the last five with Subaru/Suprime-cam. The different columns correspond to \#1: cluster ID; \#2: $d_{\mid \mathrm{WL}-\mathrm{cog} \text { (c) }}$ comoving distance between the WL peak and the BCG in kpc; \#3: $\theta_{|\mathrm{WL}-\mathrm{BCG}|}$ angular distance between the WL peak and the BCG in arcmin; \#4: $M_{200}^{\mathrm{NFW}}$ from the best NFW fit centered on the WL peak; and \#5: $M_{200}^{\mathrm{NFW}, \mathrm{BCG}}$ from the best NFW fit centered on the BCG.

about $20 \%$ when centered on the BCG for this subsample of clusters with small BCG offsets, which is within the error bars of our simulations. However, the mass difference is significantly larger for unrelaxed clusters (black dots) and can be up to $60 \%$ lower in the case of significant mergers (A851).

For about half of our sample, the BCG centering assumption would then be correct here. However, many of the clusters in this sample have significant merging activity and, therefore, the $\mathrm{BCG}$ is likely not currently the center of mass of the cluster. In addition, there are several clusters for which it is not possible to identify the BCG, and using a different center definition for these clusters would bias the mass estimate in our sample. Therefore we believe that our mass measurements are systematically high, but centering on the BCG would create masses that are systematically low, and that would not be reliable in the case of mergers, which a large number of our clusters are. A possibility would be to use the BCG center when this assumption is valid and the $\mathrm{WL}$ peak in the case of mergers, but we prefer to use the same center (WL peak) for the whole sample to be able to compare masses computed in the same way.

\section{Environment}

In this section, we use the 2D mass maps computed in Sect. 4.2 to discuss the structures detected in the vicinity of clusters. We overplot on the images the WL contours at a $3 \sigma$ significance as well as the X-ray contours and galaxy light distribution contours to fully understand the different mass components. To secure the WL detection of each structure we compute its significance level with respect to the map noise for 100 realizations of the noise. We also count the percentage of simulations in which the structure is detected at more than $3 \sigma$ above the background. The last two quantities contain similar information, and are given in Table 5. The significance levels in this table are computed from the 100 realizations of the noise and can slightly differ from the contour levels shown in Figs. 9 to 23, which correspond to the original mass maps. We also compute the number and significance of peaks expected to be due to the noise in the 
map reconstruction. This enables us to discuss the presence of WL peaks, which do not show any optical or X-ray counterpart. We also note that in the case of the optical contours, we tried to select only cluster member galaxies, while the WL is sensitive to any line-of-sight structure with a higher efficiency for structures at redshift around $z \sim 0.3-0.4$. As a result, it is not surprising to find some peaks in the convergence map with no optical counterpart.

The X-ray contours are plotted from XMM-Newton EPIC MOS1 or MOS2 images. The XMM images suit our study well, as XMM has a larger field of view than Chandra. However, when no XMM data are available, we show contours from Chandra images. Even with XMM, the field of view is limited to about 30 arcmin in diameter, and in some cases, several structures detected through weak lensing have no X-ray counterparts because only the cluster vicinity is in the X-ray field. The X-ray images have been binned in squares of 64 pixels and then smoothed with a Gaussian filter of 20 pixel width. The significance of the X-ray maps are computed from the dispersion of the values of the respective map avoiding the cluster region, and start at $2 \sigma$. We chose a $2 \sigma$ value to show better how our WL detections are embedded in the baryonic components, and because the X-ray maps are only used for qualitative description.

The light density maps are built with the galaxies selected to have a high probability of being at the same redshift as the cluster. For this, we first extract all of the objects from the images in two bands. We separate stars from galaxies and draw color-magnitude diagrams. For each cluster, we superimpose on the color-magnitude diagram the positions of the galaxies with spectroscopic redshifts coinciding with the cluster redshift range. This allows us to define the red sequence drawn by the early-type galaxies belonging to the cluster and to fit it with a linear function of the fixed slope -0.0436, as in Durret et al. (2011). We then select all the galaxies within \pm 0.3 mag of this sequence as probable cluster members and compute the density map of this galaxy catalog, using the same Gaussian kernel than that of the WL analysis. The pixel size chosen to compute these maps is $0.001 \mathrm{deg}$, and the number of bootstraps is 100 . To derive the significance level of our detections, it is necessary to estimate the mean background of each image and its dispersion. To accomplish this, we draw for each density map the histogram of the pixel intensities. We apply a $2.5 \sigma$ clipping to eliminate the pixels of the image that have high values and correspond to objects in the image. We then redraw the histogram of the pixel intensities after clipping and fit this distribution with a Gaussian. For each cluster, the mean value and width of the Gaussian, respectively, gives the mean background level and dispersion, which we call $\sigma$. We then compute the values of the contours corresponding to $3 \sigma$ detections as the background plus $3 \sigma$. In all the figures of the following subsection, we show contours starting at $3 \sigma$ and increasing by $1 \sigma$.

We first discuss the mass map of every cluster individually in Sect. 6.1, and then make general considerations in Sect. 6.2.

\subsection{Individual clusters}

In addition to discussing the reconstructed convergence maps, in this subsection we also compare the WL masses computed from the NFW best fit (see Sect. 4.3) to other masses from the literature. However, WL masses from different studies can significantly vary. The reason for that lies in the estimate of the redshift distribution of the background galaxies. In the ideal case where every study selects the same background galaxies and agrees on their redshift distribution, they should

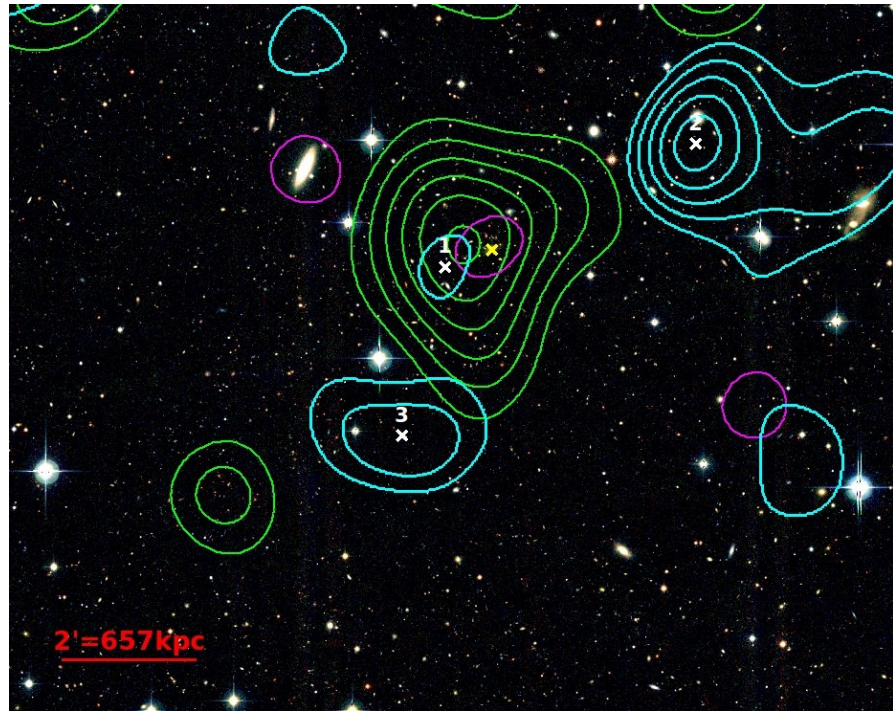

Fig. 9. Convergence density map for XDCS0329 overlaid on the threecolor CFHT/MegaCam image. Contour levels (cyan) are in signal-tonoise from $3 \sigma_{\kappa}$ with steps of $1 \sigma_{\kappa}$. Each weak lensing peak is noted as a white cross. The yellow cross indicates the position of the BCG. The X-ray contours starting at $2 \sigma_{\mathrm{X}}$ are in magenta and the light density contours starting at $3 \sigma$ are in green. We expect 1.3 fake peaks above $3 \sigma_{\kappa}$ and 0.2 above $4 \sigma_{\kappa}$ in the displayed field (see Sect. 4.2 for details). The scale is given in comoving distance.

get the same masses within errors coming just from the shear measurement. In most cases, however, the selection of galaxies and the estimate of their redshift distribution significantly vary from one study to another, introducing large differences on cluster masses. In addition, cluster masses can present a bias, for example, introduced by the choice of a given value or range of value for the concentration parameter to break the mass-concentration degeneracy. For large WL cluster surveys, masses thus differ systematically by $20-30 \%$ in comparing the masses of each cluster across the survey, however, the different teams generally agree with each other regarding which cluster are more massive.

XDCS0329, Fig. 9: XDCS0329 is barely detected, with a significance of only $2.8 \sigma_{\kappa}$. It possesses a weak X-ray and optical counterpart. A larger structure is detected at the south with WL (3: $3.9 \sigma_{\kappa}$ ) and could correspond to a structure at a different redshift from that of the cluster or to a fake peak but with a weak probability given its $\mathrm{S} / \mathrm{N}$. The most massive structure in this field lies north west of the cluster $\left(2: 5.6 \sigma_{\kappa}\right)$, and does not present any X-ray or optical detection. In addition, there is no known structure referenced at this position in NED, and its high significance detection cannot be reproduced by noise in the mass map reconstruction. A spectroscopic survey of the area would help determine the nature and redshift of this massive object. Finally, we note that XDCS0329 is a small cluster given its hydrodynamical mass of $M_{500}^{\mathrm{X}}=(2.9 \pm 0.6) \times 10^{14} M_{\odot} h_{70}^{-1}$ found in Guennou et al. (2014). It is even sometimes considered as a group rather than a cluster (e.g., Mulchaey et al. 2006).

MACSJ0454, Fig. 10: MACSJ0454 has two substructures detected in WL: a first peak at 5.1 $\sigma_{K}$, and a second at $4.2 \sigma_{K}$ defining an elongated structure, as already reported from the optical study of Kartaltepe et al. (2008). We note that these substructures are not detected in the WL reconstruction of 


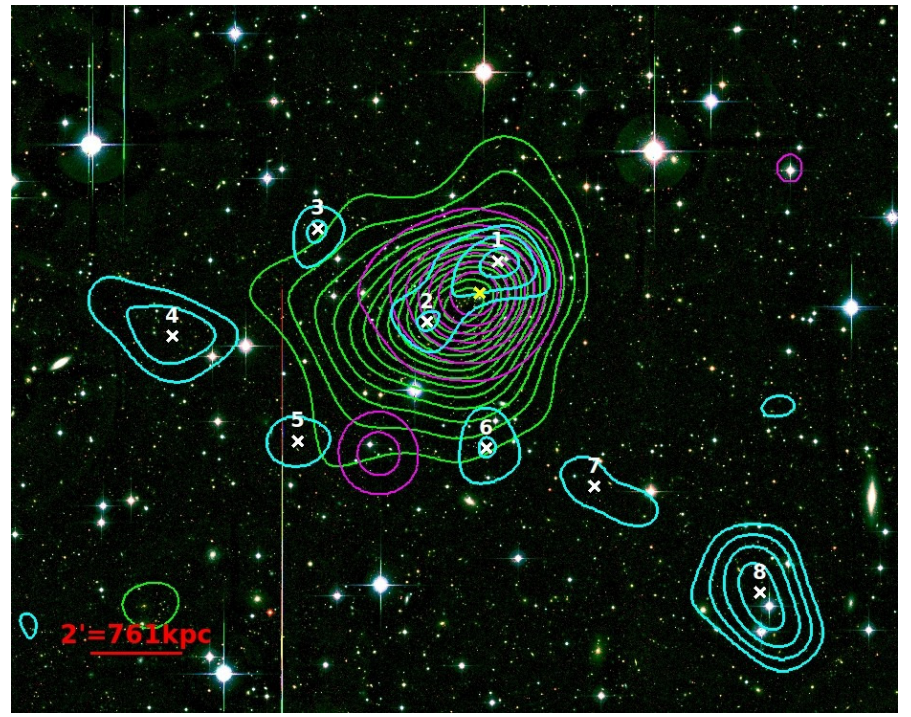

Fig. 10. Same as Fig. 9 for MACSJ0454 on the three-color CFHT/MegaCam image. We expect 3.0 fake peaks above $3 \sigma_{K}$ and 0.6 above $4 \sigma_{\kappa}$ in the displayed field (see Sect. 4.2 for details).

Soucail et al. (2015), and this is probably because they use a larger smoothing kernel $\left(\theta=150^{\prime \prime}\right.$ against $\theta=60^{\prime \prime}$ in our case). However, they found a clear elongation that matches those substructures. The X-ray and optical contours are centered between these two substructures and elongated in their direction. The fact that this cluster is highly substructured can explain why the NFW fit fails. In addition, this cluster is probably of low mass as Zitrin et al. (2011) found a central mass of $M_{500}^{\mathrm{SL}}=(0.41 \pm 0.03) \times 10^{14} M_{\odot} h_{70}^{-1}$ in their strong lensing analysis. We also detect several faint peaks. They are detected at levels of $4.4,3.8,4.2$, and $4.0 \sigma_{\kappa}$ for structures $4-7$, respectively. While structures 5 and 6 might have an optical counterparts, structure 4 and 7 very likely correspond to fake peaks or to a small group at a different redshift for structure 4 . Structures 4 and 6 are also detected in Soucail et al. (2015). A larger structure is found at the southwest ( 8 at $5.5 \sigma_{K}$ ), which is not at the cluster redshift, given that it is not detected through the galaxy density contours, but could also be due to a contamination from stars in its vicinity.

ABELL 851, Fig. 11: A851 is a massive cluster, detected at a high significance level $\left(7.6 \sigma_{K}\right)$. It is highly substructured as already found in Guennou et al. (2014) and confirmed here by the presence of three spatially separated components: dark matter, X-ray gas, and galaxies. No substructures are detected in the mass reconstruction of Soucail et al. (2015), but they used a smoothing kernel more than twice as large as ours. The most important substructures are those noted 2 and 3, the first to the south with a $5 \sigma_{\kappa}$ significance and the second to the northeast with a $4.3 \sigma_{\kappa}$ significance. These structures are also detected on the galaxy density map and perhaps also in X-rays, the contours of which are extended toward the substructure directions. Finally, we note a fourth and a fifth structures, northeast and southwest of the cluster. These are far from the cluster, and while 5 has an optical counterpart, 4 does not and could either be a fake peak or a group at a different redshift. The 5th structure should lie at the same redshift as the cluster. We note that other studies reported a higher mass than the mass we derived for this cluster. We find $M_{500}^{\mathrm{NFW}}=(4.4 \pm 1.4) \times 10^{14} M_{\odot} h_{70}^{-1}$, while Mahdavi et al. (2013)

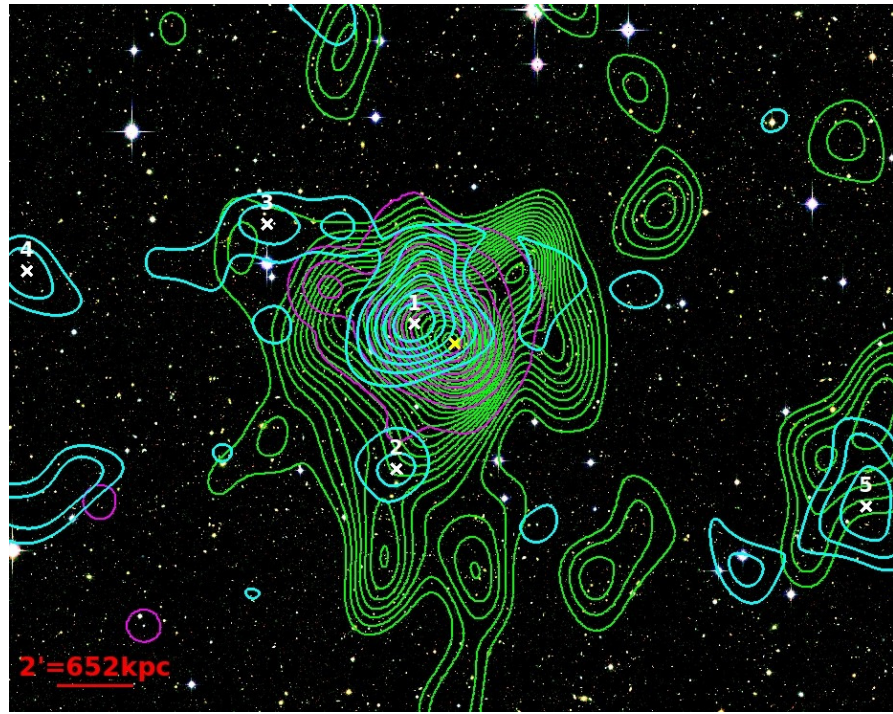

Fig. 11. Same as Fig. 9 for A851 on the three-color CFHT/MegaCam image. We expect 3.8 fake peaks above $3 \sigma_{\kappa}$ and 0.6 above $4 \sigma_{\kappa}$ in the displayed field (see Sect. 4.2 for details).

found $M_{500}=(10.5 \pm 2.5) \times 10^{14} M_{\odot} h_{70}^{-1}$ and Hoekstra et al. (2015) found $M_{500}^{\mathrm{NFW}}=(12.5 \pm 3.0) \times 10^{14} M_{\odot} h_{70}^{-1} . \mathrm{Fi}-$ nally, we note that the hydrodynamical masses from X-ray studies are lower: $M_{500}^{\mathrm{X}}=(7.4 \pm 2.3) \times 10^{14} M_{\odot} h_{70}^{-1}$ from Mahdavi et al. (2013) and $M_{500}^{\mathrm{X}}=(5.5 \pm 1.2) \times 10^{14} M_{\odot} h_{70}^{-1}$ in the present study.

LCDCS0829, Fig. 12: LCDCS0829 is at first view an isolated cluster with an elongation to the northwest. An elongation is also detected in the WL reconstruction of Soucail et al. (2015). It is detected with our three probes. However, at a larger scale there is another structure (3: $4.7 \sigma_{\kappa}$ ) about $1.5-2 \mathrm{Mpc}$ southwest from the cluster that could be in interaction and is detected both with WL and galaxy density. Farther away, but still at the same redshift according to our galaxy density map, lies a $4.5 \sigma_{\kappa}$ structure (2) that could be a group connected to the main cluster through a filamentary structure passing by 3, which remains to be detected. For this cluster, we find a mass of $M_{500}^{\mathrm{NFW}}=(5.7 \pm 2.1) \times 10^{14} M_{\odot} h_{70}^{-1}$, which agrees within the error bars with the WL study of Mahdavi et al. (2013), i.e., $M_{500}=(9.3 \pm 2.9) \times 10^{14} M_{\odot} h_{70}^{-1}$, but is low compared to that of Foëx et al. (2012), i.e., $M_{500}^{\mathrm{NFW}}=(17.7 \pm 2.2) \times 10^{14} M_{\odot} h_{70}^{-1}$.

MS1621, Fig. 13: this cluster is massive and highly substructured at large scales. The main cluster is detected at $8.3 \sigma_{K}$ and is also seen on the X-ray and galaxy density maps. It is elongated toward structures 2 and 3 detected at 4.3 and $3.5 \sigma_{\kappa}$, and also has an elongation in the X-ray and galaxy density contours for structure 2, while 3 might just be a fake peak. Finally, the galaxy density contours show a structure southeast of substructure 3 that could be a close group. Structures 1 and 2 are detected as a single structure in Soucail et al. (2015) because of the larger smoothing scale they apply to the mass map. Their reconstruction is clearly elongated in the direction of these substructures. We note that Foëx et al. (2012) found a mass of $M_{500}^{\mathrm{WL}}=(8.5 \pm 1.5) \times 10^{14} M_{\odot} h_{70}^{-1}$ that is slightly higher than our value of $M_{500}^{\mathrm{NFW}}=(6.2 \pm 1.5) \times 10^{14} M_{\odot} h_{70}^{-1}$, but in worse agreement with the hydrodynamical mass inferred from X-rays, 


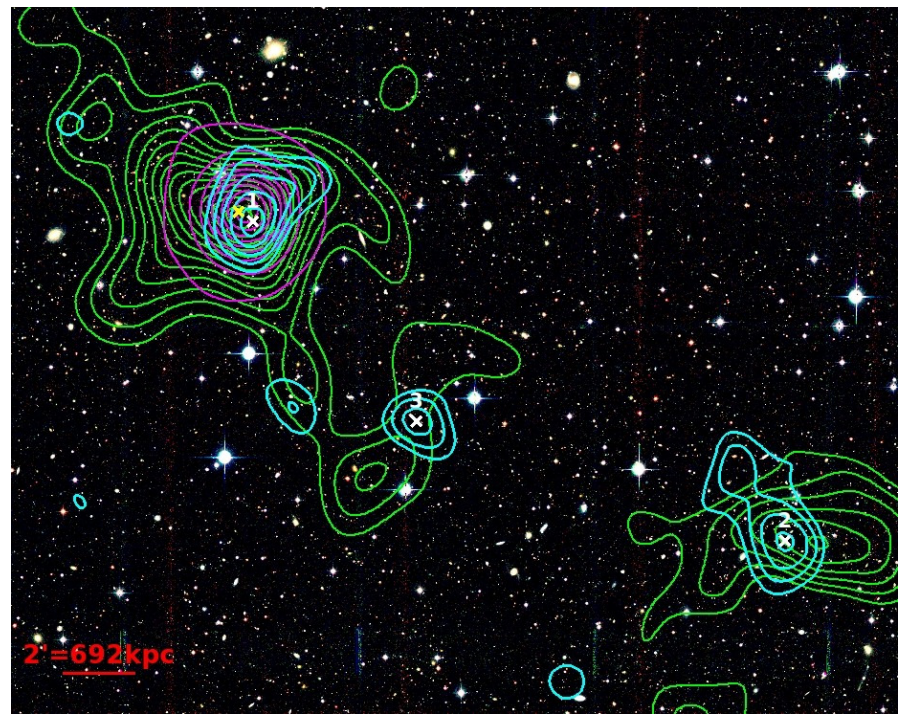

Fig. 12. Same as Fig. 9 for LCDCS0829 on a three-color CFHT/MegaCam image. We expect 4.6 fake peaks above $3 \sigma_{\kappa}$ and 0.8 above $4 \sigma_{\kappa}$ in the displayed field (see Sect. 4.2 for details).

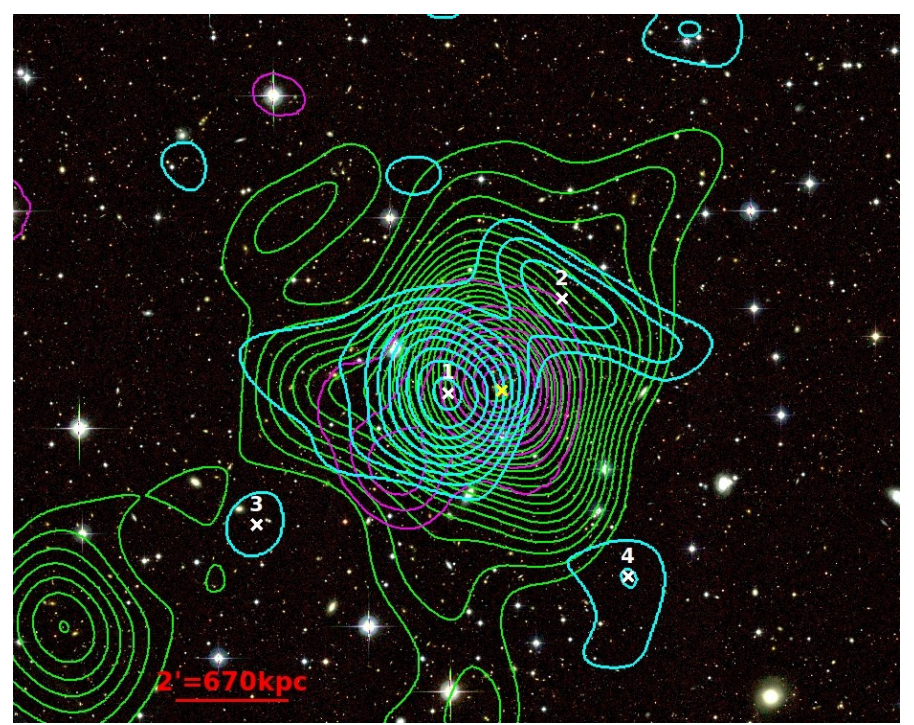

Fig. 13. Same as Fig. 9 for MS1621 on the three-color CFHT/MegaCam image. We expect 2.0 fake peaks above $3 \sigma_{\kappa}$ and 0.4 above $4 \sigma_{\kappa}$ in the displayed field (see Sect. 4.2 for details).

i.e., $M_{500}^{\mathrm{X}}=(4.5 \pm 0.5) \times 10^{14} M_{\odot} h_{70}^{-1}$.

OC02, Fig. 14: OCO2 is detected with the three probes with a $4.7 \sigma_{K}$ from WL. It seems to be merging with a smaller group on the south, detected at $4.2 \sigma_{K}$ (3). Finally, we note a massive structure detected at $5.8 \sigma_{K}$ with an X-ray counterpart and only a faint optical counterpart. This means it is a group or cluster at a different redshift from OC02. By checking on NED, we find that structure 2 in fact corresponds to Abell 2246, which is a foreground cluster at $z=0.225$. Finally, OC02, also known as CL1701+6414 is a low mass cluster. We find a mass of $M_{500}^{\mathrm{NFW}}=(2.3 \pm 1.0) \times 10^{14} M_{\odot} h_{70}^{-1}$, which is slightly higher than Israel et al. (2014), who found a WL mass of $M_{500}^{\mathrm{WL}}=0.33 \times 10^{14} M_{\odot} h_{70}^{-1}$ or $M_{500}^{\mathrm{WL}}=1.41 \times 10^{14} M_{\odot} h_{70}^{-1}$, depending on the chosen concentration parameter. We also investigate the bias in the mass estimate from the shear profile of OC02 because of the presence of the foreground cluster A2246.

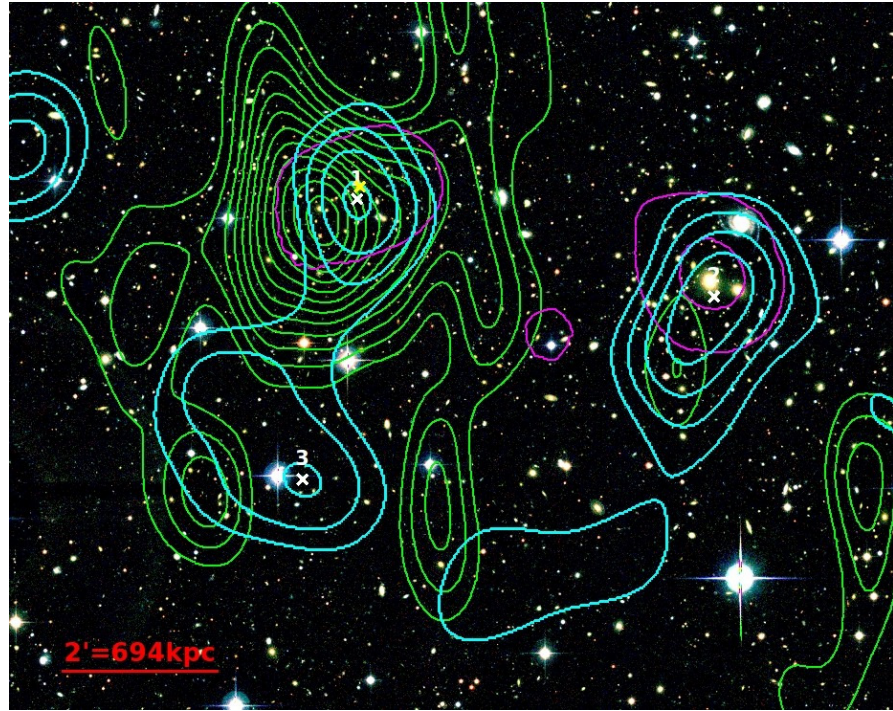

Fig. 14. Same as Fig. 9 for OC02 on the three-color CFHT/MegaCam image. We expect 1.0 fake peaks above $3 \sigma_{\kappa}$ and 0.2 above $4 \sigma_{\kappa}$ in the displayed field (see Sect. 4.2 for details).

To accomplish this, we first compute the expected shear profile for the foreground cluster, using an X-ray derived total mass from Wang \& Walker (2014) of $M_{200}^{\mathrm{X}}=(3.3 \pm 0.6) \times 10^{14} M_{\odot} h_{70}^{-1}$, and assuming a concentration parameter of $c_{200}=3.5$. We note that X-ray derived masses should not be biased by the proximity of both clusters as they are derived in a much smaller region than the WL. We then subtract this expected shear contribution to every galaxy in the field and compute the mass of OC02 again by fitting an NFW profile to its new shear profile. We find a new mass that is $7 \%$ lower than the value from Table 3 . We conclude that the presence of the foreground cluster only weakly affects the cluster mass estimate in this case, and do not correct for it as it is low compared to the other sources of error, and to avoid biasing our sample in applying a different method to one of our clusters.

NEP200, Fig. 15: NEP200 is detected in X-rays, optical, and WL with a detection significance of $5.1 \sigma_{\kappa}$. It seems to be merging with a companion on the west $\left(2: 4.6 \sigma_{\kappa}\right)$, while it is probably a projection effect given that it is not detected in the optical contours. Spectroscopic redshifts would be needed to confirm this hypothesis. We also note several peaks at $\sim 3 \sigma_{K}$ which could correspond to fake peaks or faint structures at different redshifts. As this cluster has not been widely studied yet, we derive a first WL mass of $M_{500}^{\mathrm{NFW}}=(12.7 \pm 5.5) \times 10^{14} M_{\odot} h_{70}^{-1}$ for NEP200.

RXJ2328, Fig. 16: this cluster is detected at $5.5 \sigma_{\kappa}$ from $\mathrm{WL}$ and also has X-ray and optical counterparts. From the WL contours, it seems to be merging with an infalling group detected at $3.9 \sigma_{\kappa}$ in the south. However, this structure is not detected in X-rays or in the galaxy density map, suggesting that it is at a different redshift and, therefore, is not in interaction with RXJ2328. We note the presence of the Pegasus dwarf galaxy in the south that has been masked in our analysis, but could still bias our measurements. We find a WL mass of $M_{500}^{\mathrm{NFW}}=(3.7 \pm 1.2) \times 10^{14} M_{\odot} h_{70}^{-1}$.

CLJ0152, Fig. 17: this cluster is highly substructured and has several neighboring groups nearby, implying a complex recent merging history (e.g., Massardi et al. 2010). The cluster 


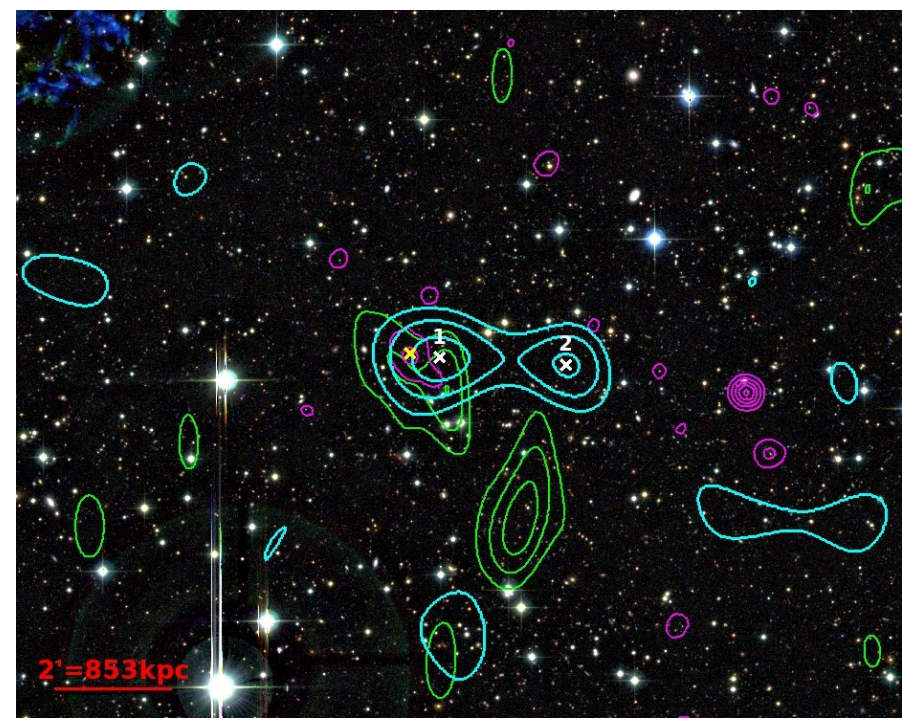

Fig. 15. Same as Fig. 9 for NEP200 on the three-color CFHT/MegaCam image. We expect 1.6 fake peaks above $3 \sigma_{\kappa}$ and 0.3 above $4 \sigma_{\kappa}$ in the displayed field (see Sect. 4.2 for details).

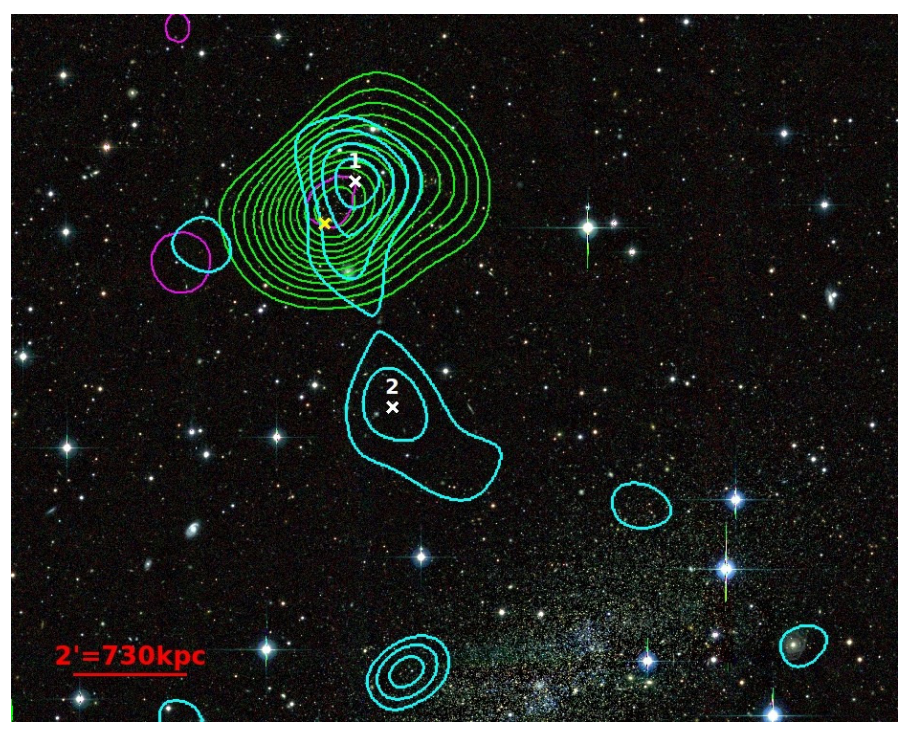

Fig. 16. Same as Fig. 9 for RXJ2328 on the three-color CFHT/MegaCam image. We expect 1.8 fake peaks above $3 \sigma_{\kappa}$ and 0.3 above $4 \sigma_{\kappa}$ in the displayed field (see Sect. 4.2 for details).

is massive $\left(M_{500}^{\mathrm{NFW}}=(9.4 \pm 3.1) \times 10^{14} M_{\odot} h_{70}^{-1}\right)$ and rather elongated in a north-south direction (see structure 2 detected at $6.4 \sigma_{K}$ ) and in a lesser extent in the east-west direction. Several structures are also detected in the south and are aligned horizontally: $3\left(4.8 \sigma_{K}\right), 4\left(6.6 \sigma_{K}\right), 5\left(4.7 \sigma_{K}\right)$, and 6 . Structures 3 and 4 , and also possibly 5, are detected in X-rays, while 4 and 6 have optical counterparts. Structures detected in WL and X-rays have a high probability to be groups, while those detected through the galaxy density maps should be around the same redshift as CLJ0152. Given the extension of the galaxy density map compared to that of the main cluster, structure 3 is probably a foreground group. One possible explanation is that the cluster recently underwent a merging event with the group 4 that passed through CLJ0152 from the northwest to the southeast. Structure 2 would be a remnant of this merging, while 3 should not have taken part in that scenario. Also structure 6 could have been created in the same event or is now interacting with structure

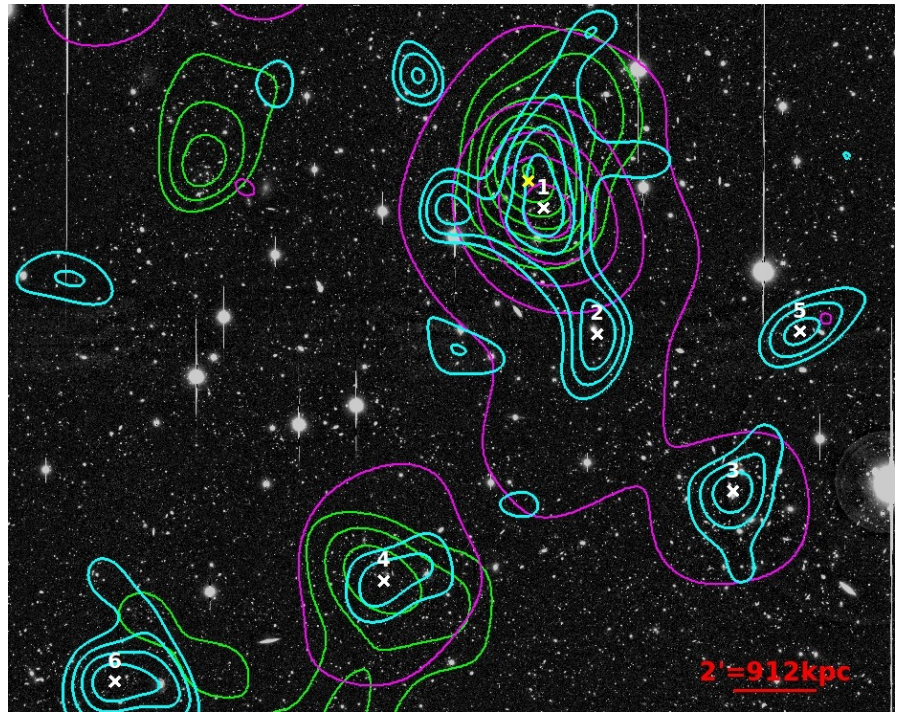

Fig. 17. Same as Fig. 9 for CLJ0152 on the $r$-band Subaru/SuprimeCam image. We expect 4.4 fake peaks above $3 \sigma_{\kappa}$ and 1.1 above $4 \sigma_{\kappa}$ in the displayed field (see Sect. 4.2 for details).

4. An X-ray temperature map would be valuable to check the direction of the past merger events.

MACSJ0717, Fig. 18: MACSJ0717 is famous for being one of the most massive clusters, as can be seen from its WL contours, which reach a significance of $10.9 \sigma_{\kappa}$. We note also that it is strongly elongated toward a southeast structure noted 2 with a $8.2 \sigma_{\kappa}$ significance. Both structures are also detected from the optical density map (as in Kartaltepe et al. 2008), suggesting that they are at the same redshift, but only the main cluster is strongly emitting in X-rays. Structure 2 is thus poor in hot gas, which makes us think that it corresponds to a filament rather than a group that would have produced more hot gas in its formation. The absence of a BCG agrees with this idea. Structure 3 could also be a continuation of this filament. This filament was first studied by Jauzac et al. (2012) from composite HST data and later by Medezinski et al. (2013). We compared our WL contours with those from Jauzac et al. (2012), and found good agreement. Concerning the mass of the cluster, Zitrin et al. (2011) and Limousin et al. (2012) found strong lensing masses of $M_{r<350 \mathrm{kpch}_{70}^{-1}}^{\mathrm{SL}}=(7.4 \pm 0.5) \times 10^{14} M_{\odot} h_{70}^{-1}$ and $M_{r<960 \mathrm{kpch}_{70}^{-1}}^{\mathrm{SL}}=(21.1 \pm 2.3) \times 10^{14} M_{\odot} h_{70}^{-1}$, respectively. From WL, various masses have been calculated in different radii. In $r_{500}$, we have a mass of $M_{500}^{\mathrm{WL}}=(15.9 \pm 4.3) \times 10^{14} M_{\odot} h_{70}^{-1}$ to be compared to Mahdavi et al. (2013) and Hoekstra et al. (2015) who respectively found $M_{500}^{\mathrm{WL}}=(16.6 \pm 3.4) \times 10^{14} M_{\odot} h_{70}^{-1}$ and $M_{500}^{\mathrm{WL}}=(22.3 \pm 5.2) \times 10^{14} M_{\odot} h_{70}^{-1}$. The first estimate is close to ours, but the second is larger and agrees only within the error bars. In a radius of $0.5 \mathrm{Mpc}$, we have $M_{r<0.5 \mathrm{Mpc} h_{70}^{-1}}^{\mathrm{WL}}=(4.4 \pm 1.2) \times 10^{14} M_{\odot} h_{70}^{-1}$, which is somewhat lower than Jauzac et al. (2012), who found a mass of $M_{r<0.53 \mathrm{Mpc} h_{70}^{-1}}^{\mathrm{WL}}=(11.0 \pm 0.8) \times 10^{14} M_{\odot} h_{70}^{-1}$. However, we find a good agreement with masses from the CLASH collaboration WL follow up (Medezinski et al. 2013), who found $M_{r<0.5 \mathrm{Mpc} h_{70}^{-1}}^{\mathrm{WL}}=(5.4 \pm 1.2) \times 10^{14} M_{\odot} h_{70}^{-1}$. Applegate et al. (2014) also found higher masses within $1.5 \mathrm{Mpc}$ with $M_{r<1.5 \mathrm{Mpc} h_{70}^{-1}}^{\mathrm{WL}}=(25.3 \pm 4.2) \times 10^{14} M_{\odot} h_{70}^{-1}$ or 


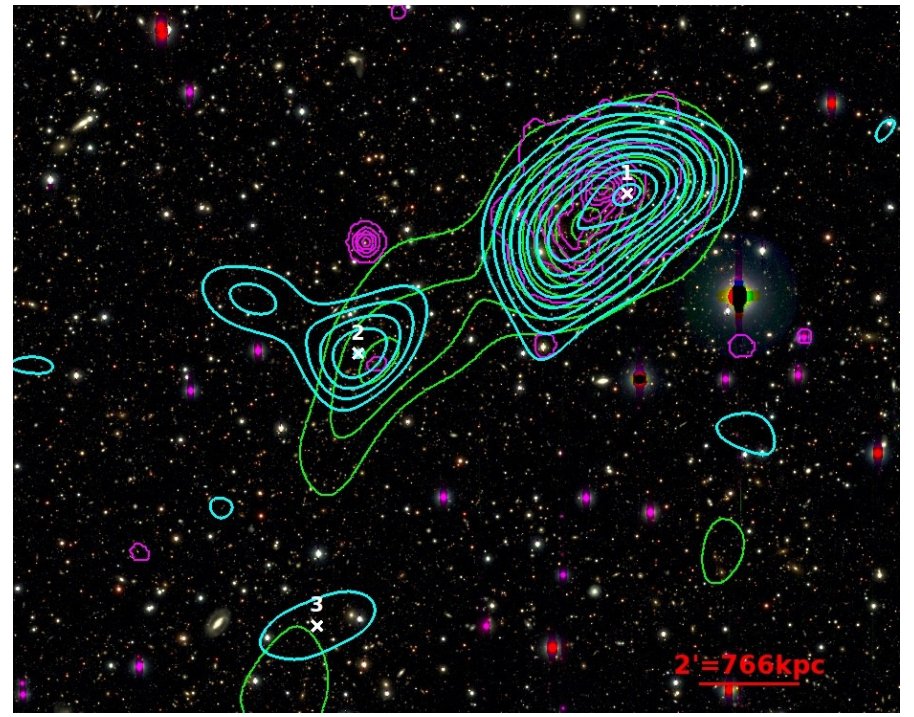

Fig. 18. Same as Fig. 9 for MACSJ0717 on the three-color Subaru/Suprime-Cam image. We expect 1.6 fake peaks above $3 \sigma_{\kappa}$ and 0.3 above $4 \sigma_{\kappa}$ in the displayed field (see Sect. 4.2 for details).

$M_{r<1.5 \mathrm{Mpc} h_{70}^{-1}}^{\mathrm{WL}}=(23.1 \pm 3.8) \times 10^{14} M_{\odot} h_{70}^{-1}$, in the first case, using the full distribution of photometric redshifts of the background galaxies and, in the second, the standard color-color cut, while we obtain $M_{r<1.5 \mathrm{Mpc} h_{70}^{-1}}^{\mathrm{WL}}=(16.1 \pm 4.5) \times 10^{14} M_{\odot} h_{70}^{-1}$. We see that the mass estimates vary strongly for this cluster; we tend to find a lower value, but in any study (including ours) MACSJ0717 appears to be one of the most massive clusters.

BMW1226, Fig. 19: this cluster is not detected through WL, probably because of its high redshift, $z=0.89$, which decreases the number of background galaxies usable for the WL reconstruction. A large elongated structure (1) is detected, however, and could be a filament linked to BMW1226. It is detected at $5.6 \sigma_{K}$ and has an optical counterpart such that is should not be too far from the cluster redshift. The small structure (2) west of the cluster is not very significant $\left(2.9 \sigma_{K}\right)$ and is probably due to the noise in the convergence map reconstruction. This cluster was studied by Jee \& Tyson (2009) under its other name, CLJ1226+3332. Using deep HST data, they manage to have a sufficient number of background galaxies to reconstruct the WL map around the cluster. However, the small field of view of the ACS camera does not allow them to study the filamentary structure that we see east of the cluster.

MACSJ1423, Fig. 20: MACSJ1423 looks rather isolated on small scales with a good alignment between the WL, X-ray, and optical centers. Kartaltepe et al. (2008) also classified it as a relaxed cluster according to its optical contours. A small structure is detected northeast from WL but not from the optical data and should correspond to a group at a different redshift. The X-ray data come from Chandra in this case, so structure 2 has no X-ray imaging. This cluster has been studied in strong lensing by Zitrin et al. (2011) and also by Limousin et al. (2010) who found a single central mass component that agrees with our smooth contours. Applegate et al. (2014) also computed WL masses for this cluster finding values of $M_{r<1.5 \mathrm{Mpc}_{70}^{-1}}^{\mathrm{WL}}=(3.7 \pm$ $2.8) \times 10^{14} M_{\odot} h_{70}^{-1}$ or $M_{r<1.5 \mathrm{Mpc} h_{70}^{-1}}^{\mathrm{WL}}=(8.8 \pm 3.6) \times 10^{14} M_{\odot} h_{70}^{-1}$, in the first case, using the full distribution of photometric redshifts

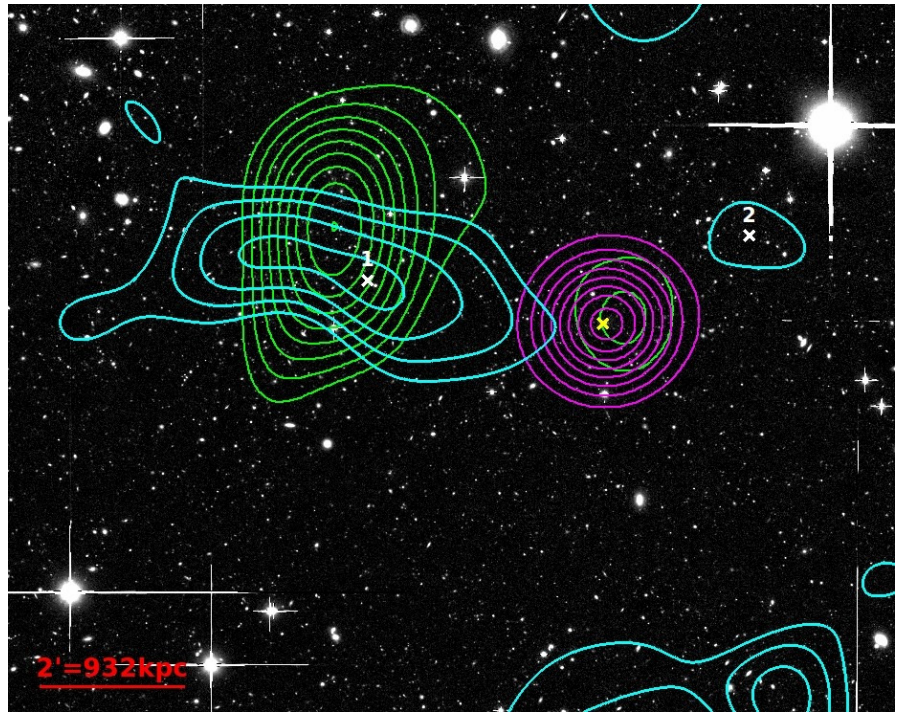

Fig. 19. Same as Fig. 9 for BMW1226 on the $r$-band Subaru/SuprimeCam image. We expect 1.0 fake peaks above $3 \sigma_{\kappa}$ and 0.1 above $4 \sigma_{\kappa}$ in the displayed field (see Sect. 4.2 for details).

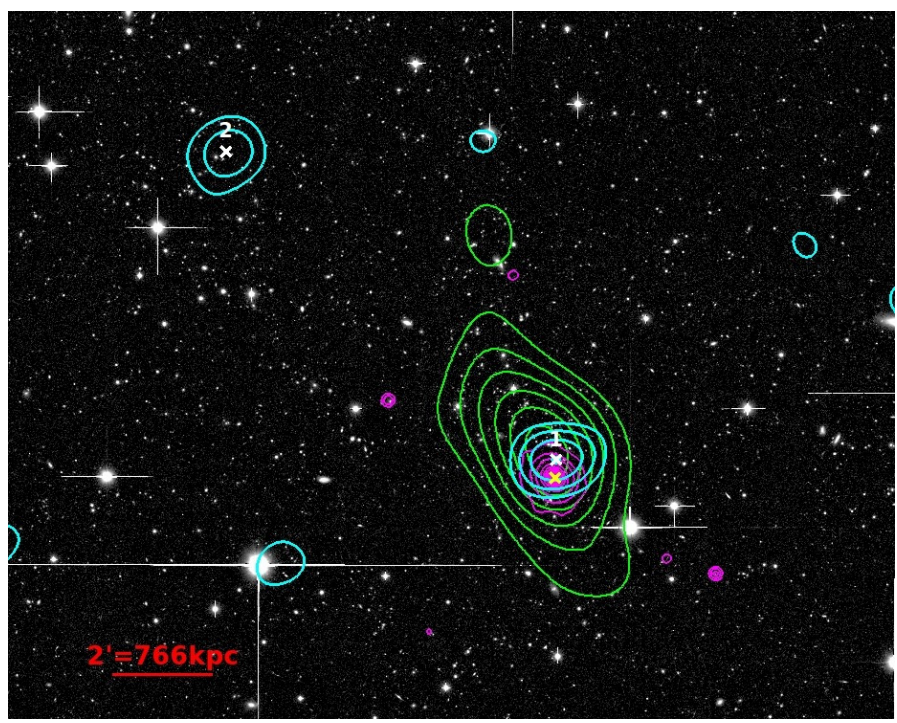

Fig. 20. Same as Fig. 9 for MACSJ1423 on the $i$-band Subaru/SuprimeCam image. We expect 2.1 fake peaks above $3 \sigma_{\kappa}$ and 0.3 above $4 \sigma_{\kappa}$ in the displayed field (see Sect. 4.2 for details).

of the background galaxies and, in the second, with the standard color-color cut. We note that our value of $M_{r<1.5 \mathrm{Mpc} h_{70}^{-1}}^{\mathrm{NFW}}=$ $(7.9 \pm 3.1) \times 10^{14} M_{\odot} h_{70}^{-1}$ is in good agreement with the one obtained with the color-color cut method that we used.

MACSJ1621, Fig. 21: MACSJ1621 presents a large substructure (2: $5.9 \sigma_{\kappa}$ significance) that could be an infalling group. Another structure (3) is detected southeast at more than $5 \sigma_{\kappa}$, and could be embedded in a filament linking it to the cluster, as suggested by the galaxy light density map. Structure 3 is also detected by von der Linden et al. (2014). An X-ray counterpart is only detected for the cluster and not for structure 2, and there is a good chance that this is part of the filament rather than an infalling group. The WL mass that we measure for this cluster agrees with the value of Applegate et al. (2014) within the error bars; we find $M_{r<1.5 \mathrm{Mpc}_{70}^{-1}}^{\mathrm{NFW}}=(5.3 \pm 1.9) \times 10^{14} M_{\odot} h_{70}^{-1}$ and they have $M_{r<1.5 \mathrm{Mpc} h_{70}^{-1}}^{\mathrm{WL}}=(8.5 \pm 2.3) \times 10^{14} M_{\odot} h_{70}^{-1}$ or 


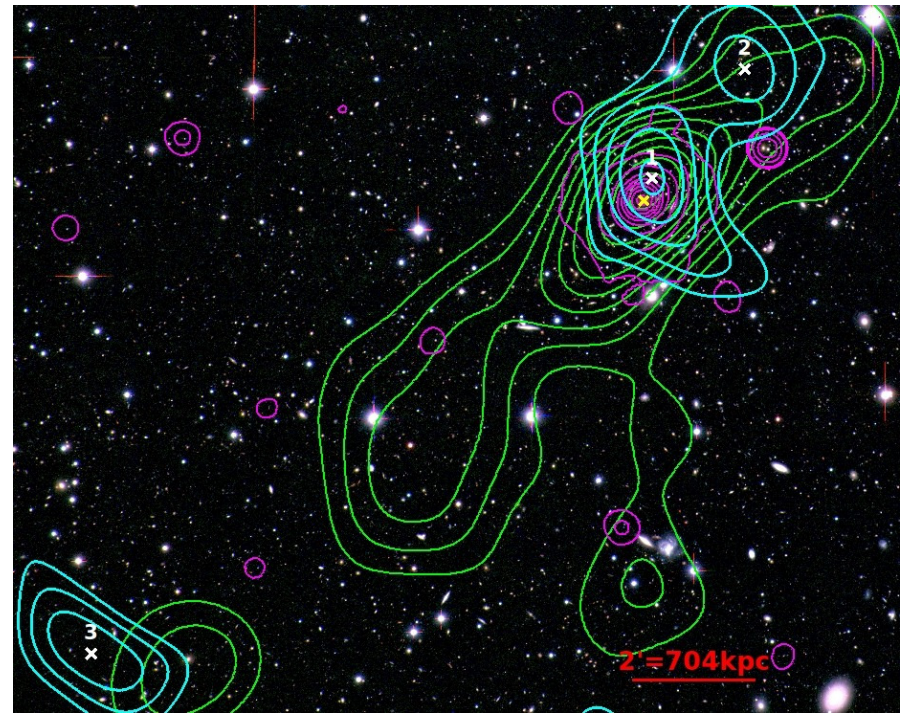

Fig. 21. Same as Fig. 9 for MACSJ1621 on the three-color Subaru/Suprime-Cam image. We expect 1.2 fake peaks above $3 \sigma_{\kappa}$ and 0.2 above $4 \sigma_{\kappa}$ in the displayed field (see Sect. 4.2 for details).

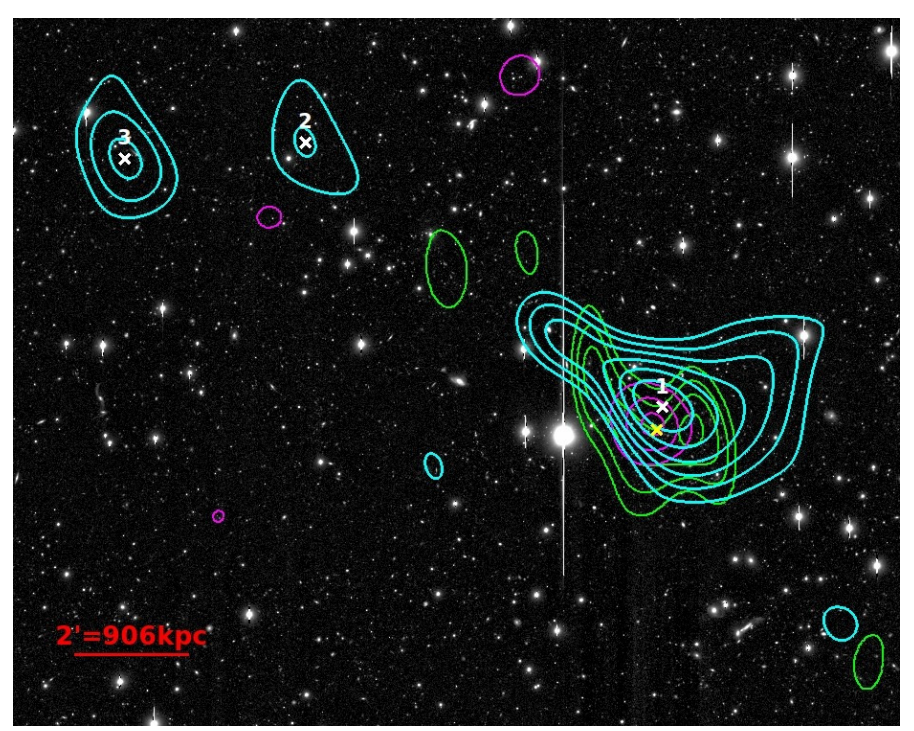

Fig. 22. Same as Fig. 9 for RXJ1716 on the $r$-band Subaru/SuprimeCam image. We expect 2.1 fake peaks above $3 \sigma_{\kappa}$ and 0.5 above $4 \sigma_{\kappa}$ in the displayed field (see Sect. 4.2 for details).

$M_{r<1.5 \mathrm{Mpc} h_{70}^{-1}}^{\mathrm{WL}}=(8.8 \pm 2.2) \times 10^{14} M_{\odot} h_{70}^{-1}$. In the first case, using the full distribution of photometric redshifts of the background galaxies and, in the second, the standard color-color cut. However, we do not reproduce the high mass found in Hoekstra et al. $(2015)$, i.e., $M_{500}^{\mathrm{NFW}}=(11.2 \pm 2.5) \times 10^{14} M_{\odot} h_{70}^{-1}$.

RXJ1716, Fig. 22: RXJ1716 $\left(1: 7.3 \sigma_{K}\right)$ shows a very elongated profile pointing toward two groups: 2 and 3 detected at 4.9 and $5.4 \sigma_{\kappa}$, respectively. However, those structures are not detected in the galaxy density map and must then lie at a different redshift. The main cluster is also detected with the $\mathrm{X}$-ray and galaxy density contours. The elongated structure to the northeast of the cluster is also seen in the WL reconstruction of Clowe et al. (1998). This is a massive cluster with $M_{500}^{\mathrm{NFW}}=(9.5 \pm 3.2) \times 10^{14} M_{\odot} h_{70}^{-1}$.

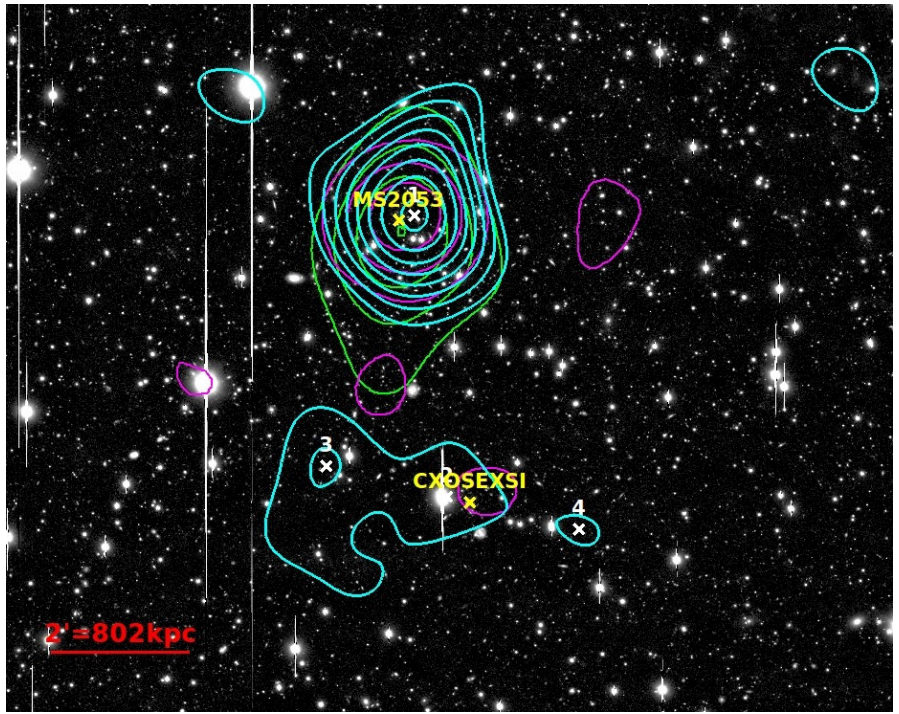

Fig. 23. Same as Fig. 9 for MS2053 and CXOSEXSI2056 on the $r$-band Subaru/Suprime-Cam image. We expect 1.3 fake peaks above $3 \sigma_{\kappa}$ and 0.2 above $4 \sigma_{\kappa}$ in the displayed field (see Sect. 4.2 for details).

MS2053/CXOSEXSI2056, Fig. 23: MS2053 is detected with a high level of significance: $8.7 \sigma_{\kappa}$ and with a mass of $M_{500}^{\mathrm{NFW}}=(6.4 \pm 2.2) \times 10^{14} M_{\odot} h_{70}^{-1}$. It is also detected in the X-ray and galaxy density contours. CXOSEXSI2056 is a smaller cluster detected at a $4.4 \sigma_{K}$ significance and also presents an X-ray counterpart. It seems to be merging with a wide structure $\left(3: 4.5 \sigma_{K}\right)$ on the east and might also be linked to the small structure 4 , but the significance of the latter structure remains low $\left(3.2 \sigma_{K}\right)$ and it is more likely a fake peak due to noise. For this field we did not try to estimate the masses of each cluster by removing the contribution from the other, as we did for $\mathrm{OC} 02$, because the significance of their detections are too different. CXOSEXSI has little chance of significantly affecting the shear profile of MS2053, and on the contrary, removing such a big cluster as MS2053 would introduce another large bias in the mass estimate of CXOSEXSI. In addition, we did not compute any mass for this latter cluster.

\subsection{General discussion}

We summarize the structure detection in Table 5, where we show the average significance of the WL detection obtained from 100 realizations of the noise along with the percentage of realizations in which the structures are detected at more than $3 \sigma$ above the map noise defined in Eq. (6). We also indicate for each structure whether it has X-ray and optical counterparts, and conclude with the current status of the cluster and possible presence of filaments.

The first conclusion from the study of this sample is that all the clusters appear very different, especially when considering their close environment. Several hypotheses made for the mass calculation are then questionable. Most of these clusters are not spherical and present either a preferential direction or several substructures. The NFW profile used in Sect. 4.3 seems simplistic compared to these results, and it appears very difficult to find a mass profile that fits every cluster, when extending to radii higher than the cluster core. 
Table 5. Results on environment.

\begin{tabular}{|c|c|c|c|c|c|c|c|}
\hline Cluster & $z$ & Structure & $\sigma_{2 \mathrm{D}}$ & $\begin{array}{l}\text { Detection } \\
\text { percentage }\end{array}$ & X-ray & Galaxies & Cluster status \\
\hline \multirow[t]{3}{*}{ XDCS0329 } & 0.4122 & 1 & 2.8 & $44 \%$ & $\mathrm{Y}$ & $\mathrm{Y}$ & - \\
\hline & & 2 & 5.6 & $96 \%$ & $\mathrm{~N}$ & $\mathrm{~N}$ & \\
\hline & & 3 & 3.9 & $74 \%$ & $\mathrm{~N}$ & $\sim$ & \\
\hline \multirow[t]{8}{*}{ MACSJ0454 } & 0.5377 & 1 & 5.1 & $91 \%$ & $\mathrm{Y}$ & $\mathrm{Y}$ & recent or present merger (3) / elongation or filament \\
\hline & & 2 & 4.2 & $76 \%$ & $\mathrm{Y}$ & $\mathrm{Y}$ & \\
\hline & & 3 & 3.7 & $67 \%$ & $\mathrm{~N}$ & $\sim$ & \\
\hline & & 4 & 4.4 & $88 \%$ & $\mathrm{~N}$ & $\mathrm{~N}$ & \\
\hline & & 5 & 3.8 & $70 \%$ & $\mathrm{~N}$ & $\sim$ & \\
\hline & & 6 & 4.2 & $82 \%$ & $\mathrm{~N}$ & $\sim$ & \\
\hline & & 7 & 4.0 & $78 \%$ & $\mathrm{~N}$ & $\mathrm{~N}$ & \\
\hline & & 8 & 5.5 & $95 \%$ & $\mathrm{~N}$ & $\mathrm{~N}$ & \\
\hline \multirow[t]{4}{*}{ ABELL851 } & 0.4069 & 1 & 7.6 & $100 \%$ & $\mathrm{Y}$ & $\mathrm{Y}$ & recent or present merger (3) / elongation or filament \\
\hline & & 2 & 5.0 & $89 \%$ & $\sim$ & $\mathrm{Y}$ & \\
\hline & & 3 & 4.3 & $81 \%$ & $\sim$ & $\mathrm{Y}$ & \\
\hline & & 4 & 4.4 & $86 \%$ & $\mathrm{~N}$ & $\mathrm{~N}$ & \\
\hline \multirow[t]{3}{*}{ LCDCS0829 } & 0.4510 & 1 & 5.5 & $98 \%$ & $\mathrm{Y}$ & $\mathrm{Y}$ & past merger (2) \\
\hline & & 2 & 4.5 & $86 \%$ & - & $\mathrm{Y}$ & \\
\hline & & 3 & 4.7 & $92 \%$ & - & $\mathrm{Y}$ & \\
\hline \multirow[t]{4}{*}{ MS1621 } & 0.4260 & 1 & 8.3 & $100 \%$ & Y & $\mathrm{Y}$ & recent or present merger (3) / elongation or filament \\
\hline & & 2 & 4.3 & $77 \%$ & $\sim$ & $\mathrm{Y}$ & \\
\hline & & 3 & 3.5 & $67 \%$ & $\mathrm{~N}$ & $\mathrm{~N}$ & \\
\hline & & 4 & 3.1 & $53 \%$ & $\mathrm{~N}$ & $\mathrm{~N}$ & \\
\hline \multirow[t]{3}{*}{$\mathrm{OC} 02$} & 0.4530 & 1 & 4.7 & $88 \%$ & $\mathrm{Y}$ & $\mathrm{Y}$ & recent or present merger (3) \\
\hline & & 2 & 5.8 & $98 \%$ & $\mathrm{Y}$ & $\sim$ & foreground cluster (A2246) \\
\hline & & 3 & 4.2 & $78 \%$ & $\mathrm{~N}$ & $\sim$ & \\
\hline \multirow{2}{*}{ NEP200 } & 0.6909 & 1 & 5.1 & $93 \%$ & Y & $\mathrm{Y}$ & recent or present merger (3) \\
\hline & & 2 & 4.6 & $84 \%$ & $\mathrm{~N}$ & $\mathrm{~N}$ & \\
\hline \multirow[t]{2}{*}{ RXJ2328 } & 0.4970 & 1 & 5.5 & $93 \%$ & $\mathrm{Y}$ & Y & recent or present merger ( 3 ) \\
\hline & & 2 & 3.9 & $73 \%$ & $\mathrm{~N}$ & $\mathrm{~N}$ & \\
\hline \multirow[t]{5}{*}{ CLJ0152 } & 0.8310 & 1 & 8.3 & $100 \%$ & $\mathrm{Y}$ & $\mathrm{Y}$ & recent or present merger (3) / elongation or filament \\
\hline & & 2 & 6.4 & $98 \%$ & $\mathrm{Y}$ & $\mathrm{N}$ & \\
\hline & & 3 & 4.8 & $85 \%$ & $\mathrm{Y}$ & $\mathrm{N}$ & \\
\hline & & 4 & 6.6 & $98 \%$ & $\mathrm{Y}$ & Y & \\
\hline & & 5 & 4.7 & $89 \%$ & $\sim$ & $\mathrm{N}$ & \\
\hline \multirow[t]{3}{*}{ MACSJ0717 } & 0.5458 & 1 & 10.9 & $100 \%$ & $\mathrm{Y}$ & $\mathrm{Y}$ & recent or present merger (3) / elongation or filament \\
\hline & & 2 & 8.2 & $100 \%$ & $\sim$ & $\mathrm{Y}$ & \\
\hline & & 3 & 5.7 & $98 \%$ & $\mathrm{~N}$ & $\mathrm{Y}$ & \\
\hline \multirow[t]{3}{*}{ BMW1226 } & 0.8900 & 0 & - & - & $\mathrm{Y}$ & $\mathrm{Y}$ & - / elongation or filament \\
\hline & & 1 & 5.6 & $97 \%$ & $\mathrm{~N}$ & $\mathrm{Y}$ & \\
\hline & & 2 & 2.9 & $46 \%$ & $\mathrm{~N}$ & $\mathrm{~N}$ & \\
\hline MACSJ1423 & 0.5450 & 1 & 5.0 & $91 \%$ & $\mathrm{Y}$ & Y & Relaxed (1) \\
\hline \multirow[t]{2}{*}{ MACSJ1621 } & 0.4650 & 1 & 6.8 & $97 \%$ & $\mathrm{Y}$ & Y & recent or present merger (3) / elongation or filament \\
\hline & & 2 & 5.9 & $96 \%$ & $\mathrm{~N}$ & $\mathrm{Y}$ & \\
\hline \multirow[t]{3}{*}{ RXJ1716 } & 0.8130 & 1 & 7.3 & $98 \%$ & $\mathrm{Y}$ & $\mathrm{Y}$ & past merger (2) \\
\hline & & 2 & 4.9 & $85 \%$ & $\mathrm{~N}$ & $\mathrm{~N}$ & \\
\hline & & 3 & 5.4 & $86 \%$ & $\mathrm{~N}$ & $\mathrm{~N}$ & \\
\hline MS2053* & 0.5830 & 1 & 8.7 & $100 \%$ & $\mathrm{Y}$ & $\mathrm{Y}$ & past merger (2) \\
\hline \multirow[t]{3}{*}{ CXOSEXSI2056* } & 0.6002 & 2 & 4.4 & $84 \%$ & $\mathrm{Y}$ & $\mathrm{N}$ & recent or present merger (3) \\
\hline & & 3 & 4.5 & $84 \%$ & $\mathrm{~N}$ & $\mathrm{~N}$ & \\
\hline & & 4 & 3.2 & $55 \%$ & $\mathrm{~N}$ & $\mathrm{~N}$ & \\
\hline
\end{tabular}

Notes. The first eight clusters are observed with CFHT/MegaCam and the last eight with Subaru/Suprime-Cam. The different columns correspond to \#1: cluster ID; \#2: cluster redshift; \#3: structure ID; \#4: significance of the WL peak in the 2D mass map in unit of $\sigma_{K}$ (see text for details); \#5: percentage of redetection above $3 \sigma_{\kappa}$ over the 100 noise realizations; \#6: detection of the structure in X-rays ( $\mathrm{Y}$ for yes, $\mathrm{N}$ for no, $\sim$ when the detection is ambiguous, - for no data); \#7: detection of the structure in galaxy density map ( $\mathrm{Y}$ for yes, $\mathrm{N}$ for no, $\sim$ when the detection is ambiguous, - for no data); and \#8: derived status of the cluster from our analysis and possible presence of a large filament; numbers refer to the classification in the text. ${ }^{(*)}$ CXOSEXSI_J205617 and MS_2053.7-0449 are on the same image. 
Despite these very different behaviors, we try to classify our sample according to the smoothness of their WL contours and the presence of substructures or infalling groups:

(1) The only relaxed cluster of our sample is MACSJ1423. On small scales we see smooth symmetrical contours and no substructures. However, even for this cluster, we find that it might be embedded in at least one filamentary structure at larger scales.

(2) The second category gathers clusters that are highly asymmetrical but do not present any clear substructure or infalling group: LCDCS0829, RXJ1716, and MS2053. These clusters are probably recovering from old merger events, of which the direction of interaction only remains visible.

(3) The last category encompasses clusters with high levels of substructuring or apparent merging events. These clusters are recovering from a recent merging event or are even presently merging. Such behaviors are observed for MACSJ0454, A851, MS1621, OC02, NEP200, RXJ2328, CLJ0152, MACSJ0717, MACSJ1621, and CXOSEXSIJ2056.

Six clusters among this last list seem to be part of particularly intense extended structures: MACSJ0454, A851, MS1621, CLJ0152, MACSJ0717, and MACSJ1621. In addition, BMW1226 shows a large filament despite the fact that the cluster is not detected itself. However, fainter elongated structures linking the different mass peaks can be seen in many cases as a result of smoothing scale $\theta_{\mathrm{S}}=1 \mathrm{arcmin}$, suggesting that every cluster lies in a large-scale structure. These LSSs are often not clearly detected, as they are too diffuse compared to the mass peaks corresponding to either infalling groups, or small merger events. Finally, we note that most of our clusters are either past mergers $(\sim 21.5 \%)$ or recent or present mergers $(\sim 71.5 \%)$. This supports the standard hierarchical scenario in which clusters grow through the merging of smaller structures. In addition, it means that most massive clusters at $0.4<z<0.9$ are still evolving through this merging process. XDCS0329 is not discussed as it is only weakly detected. This classification is summarized in Table 5.

\section{Conclusion}

We accurately measured galaxy shears for eight CFHT/ MegaCam and seven Subaru/Suprime-Cam images. We successfully estimated the mass of 12 clusters out of 16 , by fitting their shear profiles with an NFW profile. In comparison with masses from X-ray data (XMM-Newton and Chandra observations), we found that our masses are generally higher than those from X-rays by about $8 \%$; this is an expected result given that the X-ray masses rely on the hypothesis of hydrostatical equilibrium. However, our sample is small and we need higher statistics to compare both masses and also to better compare to the WL literature.

We inverted the shear to obtain convergence maps and overlaid the WL contours on images. We estimated the significance of each detected structure with 100 realizations with a random ellipticity added to each galaxy. We studied the environment of every cluster in comparison with X-ray contours and galaxy light density contours. We found that clusters are very different on large scales and doubt they can all be fitted with a simple NFW profile. We separated our sample between isolated relaxed clusters, asymmetrical clusters with no substructures and clusters that have a more complex environment. The second category corresponds to past mergers and the third to recent or present mergers. Most of the sampled clusters are in the last two categories, providing strong observational support to the hierarchical growth scenario, and implying that clusters are still evolving through this process at $0.4<z<0.9$. Temperature maps from deep X-ray imaging could help characterize the different merging phases that we observe (see, e.g., Durret et al. 2011, and references therein). Even in the isolated case, we found that clusters are embedded in complex large-scale structures, often connecting to another group on megaparsec scales. We report possible filament detections in CLJ0152, MACSJ0454, MACSJ0717, A851, BMW1226, MS1621, and MACSJ1621, the first one also experiencing recent complex merger events. Finally, it is important to note that the distinction between a filament and an infalling group or small cluster is almost a semantic problem. However, groups and small clusters should contain more X-ray gas than filaments and are more likely to possess a BCG, at least in the case of clusters. A more detailed study of each cluster with separate simulations is required to help distinguish between the two possibilities. We intend to study the galaxy populations of the proposed filaments in the framework of the DAFT/FADA survey, a work that will also help in discriminating the nature of these structures.

Acknowledgements. We thank Raphael Gavazzi and Hugo Capelato for useful discussions and Mathilde Jauzac for sharing her WL contours of MACSJ0717 with us. We are grateful to the anonymous referee for his/her careful reading and comments that improved the quality of the manuscript. We thank Nick Kaiser for authorizing us to use the IMCAT software, Emmanuel Bertin for making his astromatic softwares publicly available, and Martin Kilbinger for his ATHENA software. D.C. acknowledges support from the National Science Foundation under Grant No. 1109576. F.D. acknowledges long-term support from CNES. I.M. acknowledges financial support from the Spanish Ministry of Economy and Competitiveness through the grant AYA2012-42227P.

\section{References}

Allen, S. W., Evrard, A. E., \& Mantz, A. B. 2011, ARA\&A, 49, 409 Applegate, D. E., von der Linden, A., Kelly, P. L., et al. 2014, MNRAS, 439, 48 Bahé, Y. M., McCarthy, I. G., \& King, L. J. 2012, MNRAS, 421, 1073 Bartelmann, M., \& Schneider, P. 2001, Phys. Rep., 340, 291

Battaglia, N., Bond, J. R., Pfrommer, C., \& Sievers, J. L. 2013, ApJ, 777, 123 Becker, M. R., \& Kravtsov, A. V. 2011, ApJ, 740, 25

Bertin, E. 2006, in Astronomical Data Analysis Software and Systems XV, eds. C. Gabriel, C. Arviset, D. Ponz, \& S. Enrique, ASP Conf. Ser., 351, 112

Bertin, E. 2011, in Astronomical Data Analysis Software and Systems XX, eds. I. N. Evans, A. Accomazzi, D. J. Mink, \& A. H. Rots, ASP Conf. Ser., 442, 435

Bertin, E., \& Arnouts, S. 1996, A\&AS, 117, 393

Bertin, E., Mellier, Y., Radovich, M., et al. 2002, in Astronomical Data Analysis Software and Systems XI, eds. D. A. Bohlender, D. Durand, \& T. H. Handley, ASP Conf. Ser., 281, 228

Bond, J. R., Kofman, L., \& Pogosyan, D. 1996, Nature, 380, 603

Bruzual, G., \& Charlot, S. 2003, MNRAS, 344, 1000

Chabrier, G. 2003, PASP, 115, 763

Clowe, D., \& Schneider, P. 2001, A\&A, 379, 384

Clowe, D., Luppino, G. A., Kaiser, N., Henry, J. P., \& Gioia, I. M. 1998, ApJ, 497, L61

Clowe, D., Schneider, P., Aragón-Salamanca, A., et al. 2006, A\&A, 451, 395

Clowe, D., Markevitch, M., Bradač, M., et al. 2012, ApJ, 758, 128

Cypriano, E. S., Sodré, Jr., L., Kneib, J.-P., \& Campusano, L. E. 2004, ApJ, 613, 95

Dahle, H., Kaiser, N., Irgens, R. J., Lilje, P. B., \& Maddox, S. J. 2002, ApJS, 139,313

Diemer, B., \& Kravtsov, A. V. 2014, ApJ, 789, 1

Dietrich, J. P., Schneider, P., Clowe, D., Romano-Díaz, E., \& Kerp, J. 2005, A\&A, 440, 453

Dietrich, J. P., Werner, N., Clowe, D., et al. 2012, Nature, 487, 202

Durret, F., Laganá, T. F., \& Haider, M. 2011, A\&A, 529, A38

Foëx, G., Soucail, G., Pointecouteau, E., et al. 2012, A\&A, 546, A106

Gao, L., Navarro, J. F., Cole, S., et al. 2008, MNRAS, 387, 536

Gavazzi, R., \& Soucail, G. 2007, A\&A, 462, 459 
Gavazzi, R., Mellier, Y., Fort, B., Cuillandre, J.-C., \& Dantel-Fort, M. 2004, A\&A, 422, 407

Gray, M. E., Taylor, A. N., Meisenheimer, K., et al. 2002, ApJ, 568, 141

Guennou, L., Adami, C., Ulmer, M. P., et al. 2010, A\&A, 523, A21

Guennou, L., Adami, C., Durret, F., et al. 2014, A\&A, 561, A112

Heymans, C., Gray, M. E., Peng, C. Y., et al. 2008, MNRAS, 385, 1431

Hoekstra, H. 2007, MNRAS, 379, 317

Hoekstra, H., Franx, M., Kuijken, K., \& Squires, G. 1998, ApJ, 504, 636

Hoekstra, H., Herbonnet, R., Muzzin, A., et al. 2015, MNRAS, 449, 685

Ilbert, O., Capak, P., Salvato, M., et al. 2009, ApJ, 690, 1236

Israel, H., Reiprich, T. H., Erben, T., et al. 2014, A\&A, 564, A129

Jain, B., \& Taylor, A. 2003, Phys. Rev. Lett., 91, 141302

Jauzac, M., Jullo, E., Kneib, J.-P., et al. 2012, MNRAS, 426, 3369

Jee, M. J., \& Tyson, J. A. 2009, ApJ, 691, 1337

Kaiser, N. 2011, Astrophysics Source Code Library

[record ascl:1108.001]

Kaiser, N., \& Squires, G. 1993, ApJ, 404, 441

Kaiser, N., Squires, G., \& Broadhurst, T. 1995, ApJ, 449, 460

Kaiser, N., Wilson, G., Luppino, G., et al. 1998, ArXiv e-prints [arXiv: astro-ph/9809268]

Kartaltepe, J. S., Ebeling, H., Ma, C. J., \& Donovan, D. 2008, MNRAS, 389, 1240

Kilbinger, M., Bonnett, C., \& Coupon, J. 2014, Astrophysics Source Code Library [record ascl: 1402.026]

Kravtsov, A. V., Vikhlinin, A., \& Nagai, D. 2006, ApJ, 650, 128

Laganá, T. F., Martinet, N., Durret, F., et al. 2013, A\&A, 555, A66

Limousin, M., Ebeling, H., Ma, C.-J., et al. 2010, MNRAS, 405, 777

Limousin, M., Ebeling, H., Richard, J., et al. 2012, A\&A, 544, A71

Luppino, G. A., \& Kaiser, N. 1997, ApJ, 475, 20

Mahdavi, A., Hoekstra, H., Babul, A., et al. 2013, ApJ, 767, 116

Mancone, C. L., \& Gonzalez, A. H. 2012, PASP, 124, 606

Martinet, N., Bartlett, J. G., Kiessling, A., \& Sartoris, B. 2015a, A\&A, 581, A101

Martinet, N., Durret, F., Guennou, L., et al. 2015b, A\&A, 575, A116

Massardi, M., Ekers, R. D., Ellis, S. C., \& Maughan, B. 2010, ApJ, 718, L23

Massey, R., Refregier, A., Bacon, D. J., Ellis, R., \& Brown, M. L. 2005, MNRAS, 359, 1277
Massey, R., Heymans, C., Bergé, J., et al. 2007a, MNRAS, 376, 13

Massey, R., Rhodes, J., Ellis, R., et al. 2007b, Nature, 445, 286

Maughan, B. J., Giles, P. A., Randall, S. W., Jones, C., \& Forman, W. R. 2012, MNRAS, 421, 1583

Mead, J. M. G., King, L. J., \& McCarthy, I. G. 2010, MNRAS, 401, 2257

Medezinski, E., Umetsu, K., Nonino, M., et al. 2013, ApJ, 777, 43

Meneghetti, M., Rasia, E., Vega, J., et al. 2014, ApJ, 797, 34

Mulchaey, J. S., Lubin, L. M., Fassnacht, C., Rosati, P., \& Jeltema, T. E. 2006, ApJ, 646, 133

Murphy, K. J., Schrabback, T., Clowe, D., et al. 2015, A\&A, submitted

Nagai, D., Vikhlinin, A., \& Kravtsov, A. V. 2007, ApJ, 655, 98

Navarro, J. F., Frenk, C. S., \& White, S. D. M. 1996, ApJ, 462, 563

Oguri, M., Takada, M., Okabe, N., \& Smith, G. P. 2010, MNRAS, 405, 2215

Okabe, N., \& Smith, G. P. 2015, MNRAS, submitted [arXiv: 1507.04493]

Okabe, N., Takada, M., Umetsu, K., Futamase, T., \& Smith, G. P. 2010, PASJ, 62,811

Rasia, E., Ettori, S., Moscardini, L., et al. 2006, MNRAS, 369, 2013

Schneider, P., King, L., \& Erben, T. 2000, A\&A, 353, 41

Schneider, P., van Waerbeke, L., Kilbinger, M., \& Mellier, Y. 2002, A\&A, 396,

Seitz, C., \& Schneider, P. 1995, A\&A, 297, 287

Smail, I., Ellis, R. S., Fitchett, M. J., \& Edge, A. C. 1995, MNRAS, 273, 277

Soucail, G., Foëx, G., Pointecouteau, E., Arnaud, M., \& Limousin, M. 2015, A\&A, 581, A31

Springel, V., White, S. D. M., Jenkins, A., et al. 2005, Nature, 435, 629

Tegmark, M., Blanton, M. R., Strauss, M. A., et al. 2004, ApJ, 606, 702

Umetsu, K., Broadhurst, T., Zitrin, A., et al. 2011, ApJ, 738, 41

van Waerbeke, L. 2000, MNRAS, 313, 524

von der Linden, A., Allen, M. T., Applegate, D. E., et al. 2014, MNRAS, 439, 2

Wang, Q. D., \& Walker, S. 2014, MNRAS, 439, 1796

Wright, C. O., \& Brainerd, T. G. 2000, ApJ, 534, 34

Zitrin, A., Broadhurst, T., Barkana, R., Rephaeli, Y., \& Benítez, N. 2011, MNRAS, 410, 1939 


\section{Appendix A: Validating the PSF correction}

In order to validate our PSF correction, we compute the autocorrelation functions of star ellipticities before and after correcting for the PSF (Fig. A.1), and we also compare the autocorrelation function of the shear to the cross-correlation function between the galaxy-corrected shears and the stellar ellipticities before correction (Fig. A.2). Results are shown for MACSJ0717 and RXJ2328, which correspond to the Subaru/Suprime-Cam and CFHT/Megacam data, respectively . The correlation functions are computed using the ATHENA software (Kilbinger et al. 2014; Schneider et al. 2002), from a 1 arcmin separation angle to 30 arcmin. $C_{1}$ and $C_{2}$ correspond to the rotated 1 and 2 components, i.e., when taking the correlation between a given pair, $C_{1}$ compares the shear that is tangential to the line connecting the pairs and $C_{2}$ is the 45 degree component.
Figure A.1 shows that the PSF correction has reduced the star ellipticity auto-correlation function by about three orders of magnitude both for the Suprime-cam and Megacam data. In addition, we see in Fig. A. 2 that the correlation between shear and stars is consistent with zero and, thus, that the residual bias from the PSF correction does not significantly affect the shear, which shows classical auto-correlation functions.

\section{Appendix B: Shear profiles}

In this section we present the shear profiles for every cluster. See Sect. 4.3 for details about how shear profiles are computed and how the NFW fit is performed. 
N. Martinet et al.: Weak lensing study around high redshift clusters
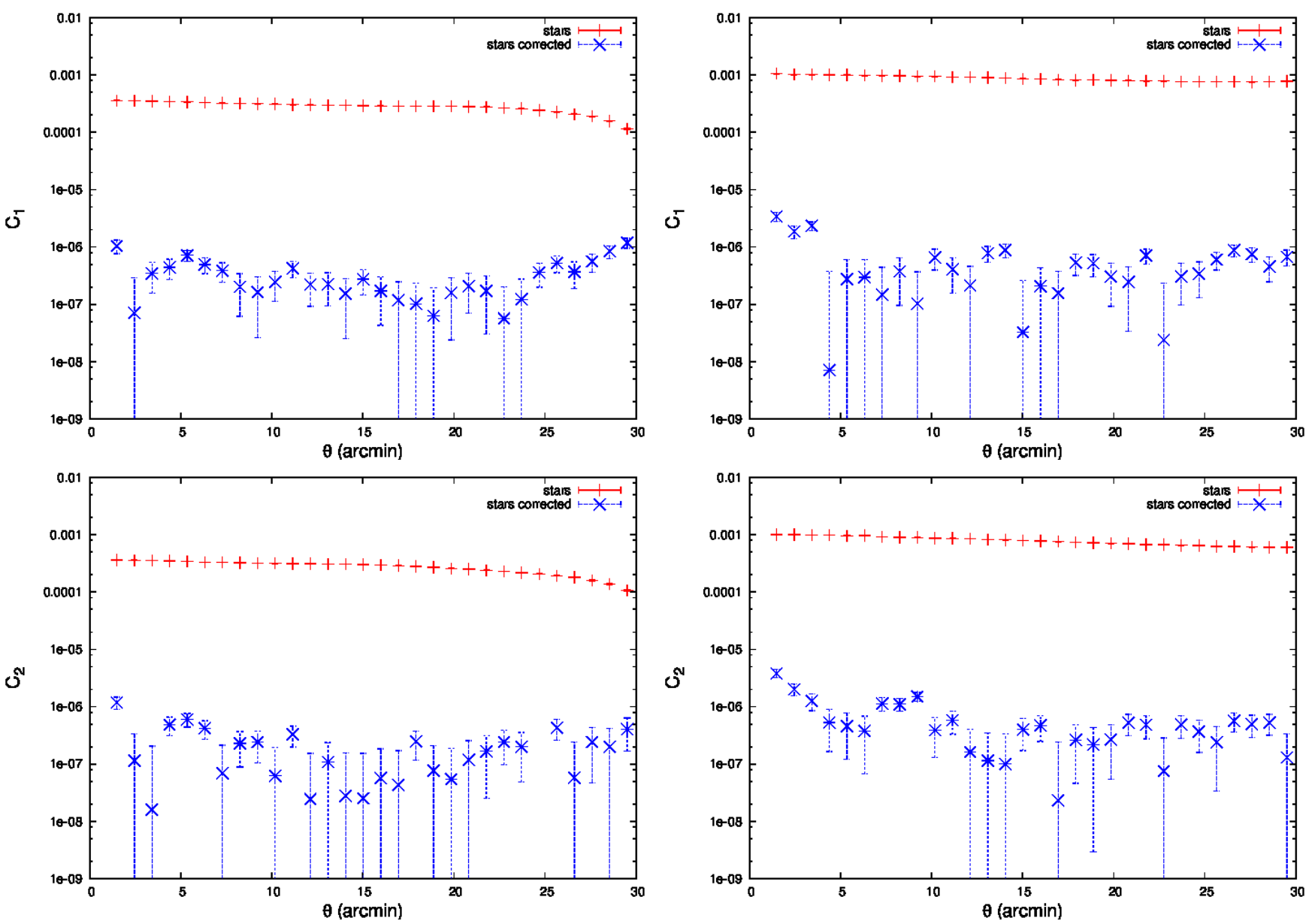

Fig. A.1. Correlation functions of stellar ellipticities before (red) and after correcting for the PSF (blue) for a Subaru/Suprime-Cam field (MACSJ0717) on the left and a CFHT/Megacam field (RXJ2328) on the right. For the corrected auto-correlation function, we plot the absolute values while the true values fluctuate around zero because negative values are not well-displayed in logscale. 
A\&A 590, A69 (2016)
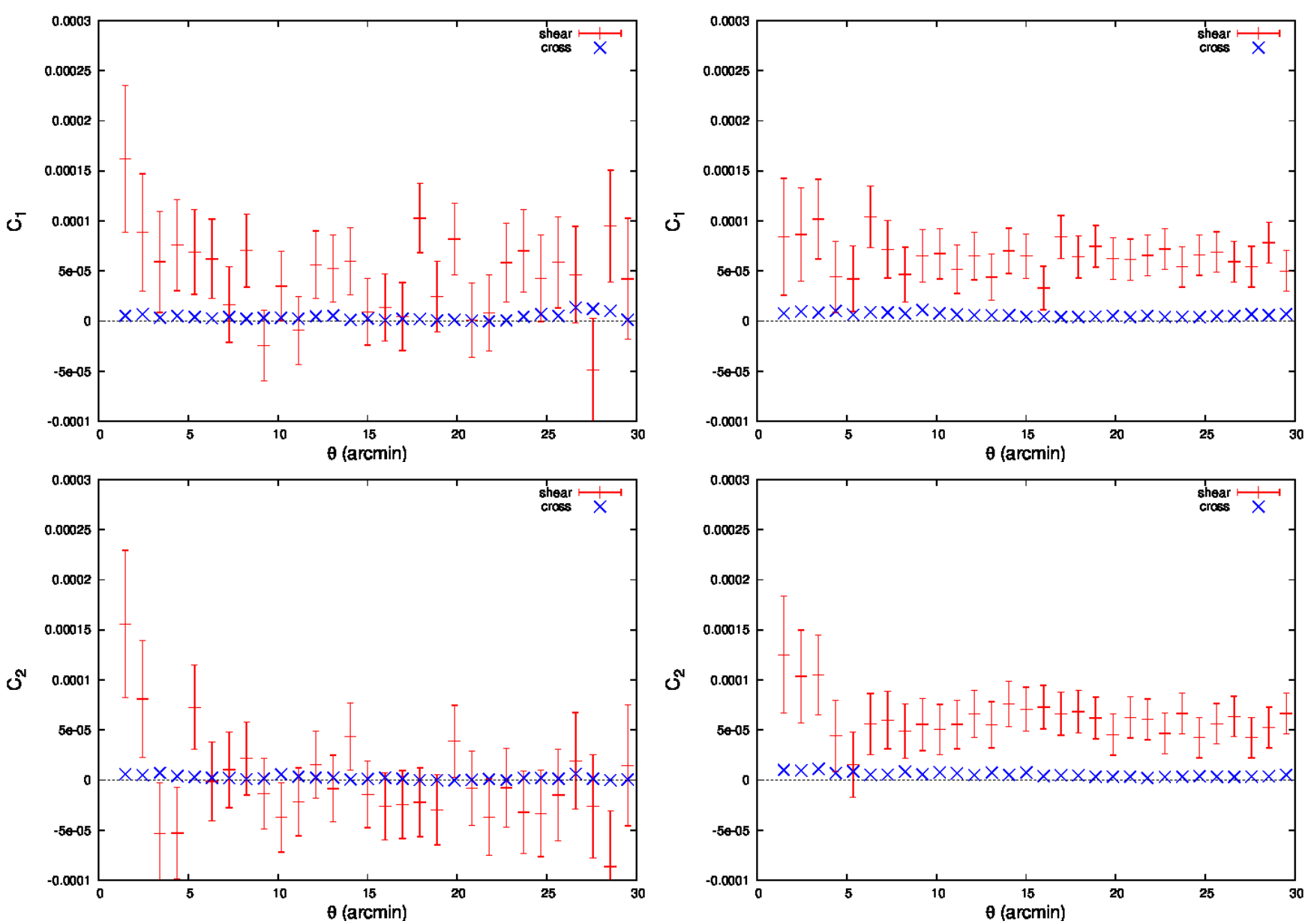

Fig. A.2. Correlation function of the shear (red) and cross -correlation function between the shear and the uncorrected star ellipticity (blue) for a Subaru/Suprime-Cam field (MACSJ0717) on the left and a CFHT/Megacam field (RXJ2328) on the right. 
N. Martinet et al.: Weak lensing study around high redshift clusters
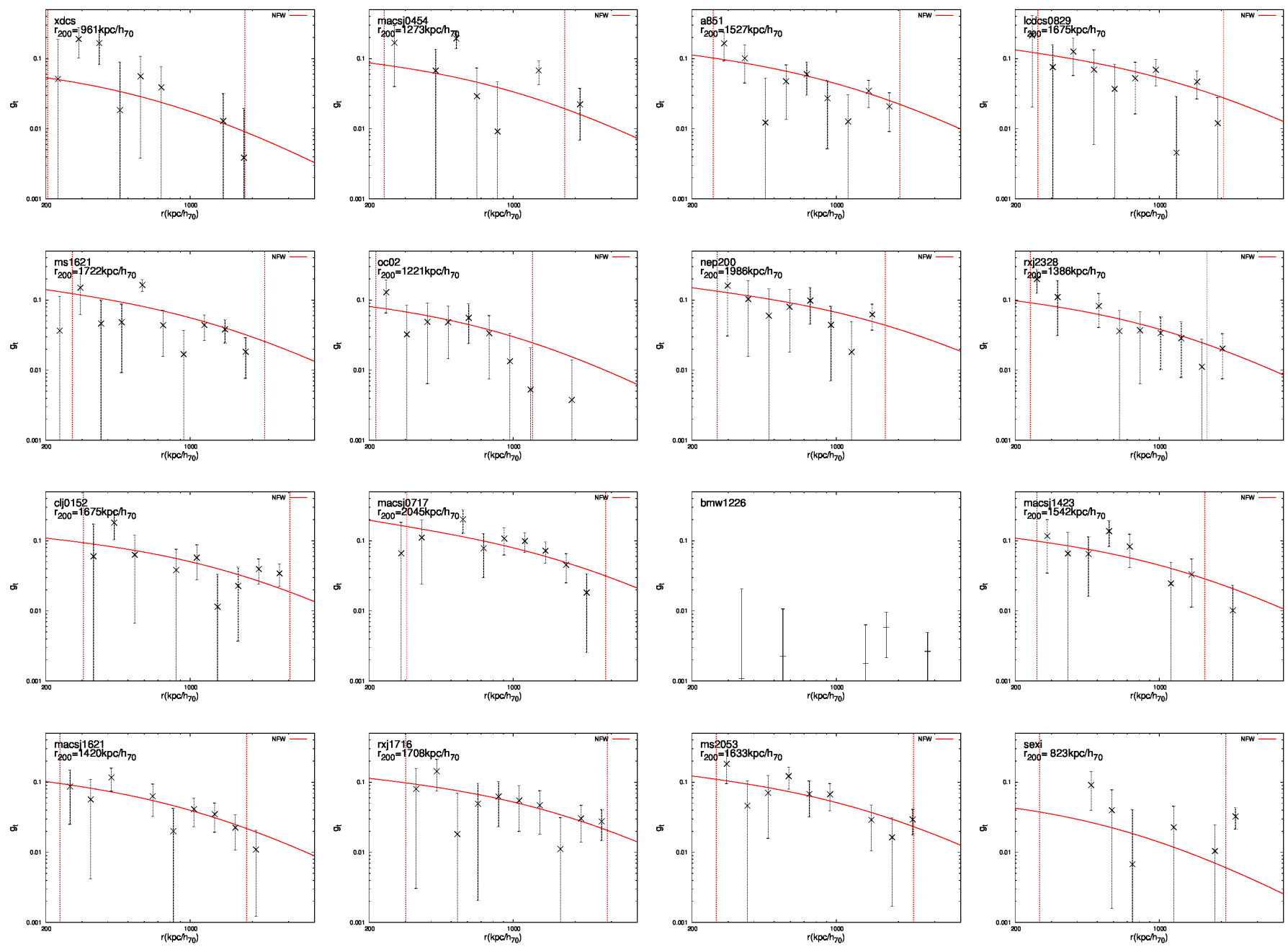

Fig. B.1. Tangential shear profile (black points) and best NFW fit (red curve) for every cluster centered on the WL peak. The error bars correspond to the rotated shear and should be equal to zero in the absence of noise. The red dotted lines represent the inner and outer radii used for the fit. See Sect. 4.3 for details. The $r_{200}$ values shown can slightly differ from Table 3 because they are calculated for a single fit to the data, while we show the mean over 100 realizations of the noise in Table 3. We note that clusters with a low significance fit have most of their shear profile compatible with zero (XDCS0329, MACSJ0454, BMW1226, CXOSEXSI2056). 